\title{
Final Technical Report on Scaling Models of the Internal Variability of Clouds DoE Grant No. DE-FG02-04ER63773
}

\author{
K. Ivanova \\ Department of Meteorology, The Pennsylvania State University \\ University Park, PA 16802, USA
}

April 24, 2008

\begin{abstract}
The purpose of this proposal is to gain a better understanding of the space-time correlations of atmospheric fluctuations in clouds through application of methods from statistical physics to high resolution, continuous data sets of cloud observations available at the Department of Energy Atmospheric Radiation Measurement Program archive. In this report we present the accomplishments achieved during the four year period. Starting with the most recent one, we report on two breakthroughs in our research that make the fourth year of the project exceptionally successful and markedly outperforming the objectives. The first break-through is on characterization of the structure of cirrus radiative properties at large, intermediate and small, generating cells scales by applying the Fokker-Planck equation method and other methods to ARM millimeter wavelength radar observations collected at the Southern Great Plains site. The second break-through is that we show that different characterizations of the cirrus radiative properties are obtained for different synoptic scale environments. We outline a stochastic approach to investigate the internal structure of radiative properties of cirrus clouds based on empirical modeling and draw conclusions about cirrus dynamical properties in the context of the synoptic environment. Results on the structure of cirrus dynamical properties are consistent with the structure of cirrus based on aircraft in situ measurements, with results from ground-based Raman lidar, and with results from model studies. These achievements would not have been possible without the accomplishments from the previous years on a number of problems that involve application of methods of analysis such as the Fokker-Planck equation approach, Tsallis nonextensive statistical mechanics, detrended fluctuation analysis, and others. These include stochastic analysis of neutrally stratified cirrus layers, internal variability and turbulence in cirrus, dynamical model and nonextensive statistical mechanics of liquid water path fluctuations, etc.
\end{abstract}




\section{Goals and Objectives}

The overall objective of our project is to gain a better understanding of the spacetime correlations of atmospheric fluctuations in clouds. To achieve this goal we apply methods and models from statistical physics to high quality, continuous data sets of cloud observations available at the ARM archive.

Summary of Deliverables: (listed on p. 40)

- 4 Peer-Reviewed Journal Papers

- 6 Papers Published in Conference Proceedings

- 10 Poster Presentations at International Conferences

- 2 Talks Presented at International Conferences

\section{Summary of Accomplishments:}

- Tracking nucleation-growth-sublimation in cirrus clouds using ARM millimeter wavelength radar observations (Ivanova and Ackerman, 2008a,b) (p. 4)

Two break-throughs in our research make the fourth year of the project exceptionally successful and markedly outperforming the objectives. The first break-through is on characterization of the structure of cirrus radiative properties at large, intermediate and small, generating cells scales by applying the Fokker-Planck equation (FPE) method and other methods to ARM millimeter wavelength radar (MMCR) observations collected at the Southern Great Plains (SGP) site. The second breakthrough is that we show that different characterizations are obtained for different synoptic scale environments.

- Stochastic analysis of neutrally stratified cirrus layer (Ivanova and Ackerman, 2007a) (p. 30)

Neutrally stratified layers of cirrus clouds are known to be associated with the existence of generating cells. We quantify the structure of the cloud-generating layer in cirrus observed at the ARM SGP site through empirical analysis of radar reflectivity observations based on the FPE method.

- An attempt to distinguish dynamical and microphysical contributions to the radiative properties of cirrus clouds at the ARM SGP site (Ivanova and Ackerman, 2007b). (p. 30)

The problem of unraveling the interwoven dynamical, microphysical and radiative properties in cirrus has been of interest to the research community for many years. We address this problem through analysis of radar reflectivity observations from cirrus clouds collected at the ARM SGP site.

- Atmospheric turbulence as a Markov process (Ivanova and Ackerman, 200\%c) (p. 31)

Results from this research with emphasis on the relationship between the results 
from empirical analysis of cirrus radiative properties observed at the ARM SGP site and physical processes that are responsible for producing such properties, were presented at the 23rd International Conference on Statistical Physics of the International Union for Pure and Applied Physics (IUPAP).

- Dynamical model and nonextensive statistical mechanics of liquid water path fluctuations in stratus clouds (Ivanova et al., 2007) (p. 33)

Dynamical model of liquid water path fluctuations in stratus clouds observed at the ARM SGP site is suggested within the framework of the non-extensive statistical mechanics. We applied the recently suggested Beck model of hydrodynamic turbulence to describe and model probability distribution functions of liquid water path in stratus clouds.

- Internal variability and turbulence in cirrus: Fokker-Planck equation approach (Ivano-va 2006, Ivanova et al., 2006a,b). (p. 34)

We obtain and analyze the time-dependent probability distribution functions of time series of radar reflectivity measurements in cirrus observed at the ARM SGP site. We show that regions having different thermodynamic stratification that are apparently dominated by different physical processes, including turbulence can be distinguished as a result of the analysis.

- Internal variability dependence in cirrus cloud structure (Ivanova et al., 2005a,b) (p. 35)

We report results on distinguishing various vertical regions in the cloud that are apparently dominated by different physical phenomena.

- Time-dependent distribution of liquid water path in stratus clouds (Ivanova, 2004, Ivanova et al., 2004b). (p. 36)

The LWP signal is non-stationary with highly irregular and clustered fluctuations owing to a set of various physical influences that occur over different temporal and spatial scales. Thus, it is of interest to both distinguish and quantify from first principles the deterministic and stochastic influences on the LWP signal in stratus clouds.

- Local correlations and multifractal behavior in marine boundary layer cloud dynamics (Kitova et al., 2004). (p. 37)

We present an analysis of the local correlations and the multi-affine properties of cloud base height with respect to their diurnal evolution.

- Time correlations in cirrus clouds and internal variability at isodepths of the cloud (Ivanova et al., 2004a). (p. 38)

We first focused our efforts on answering the question of whether or not the parameters characterizing the time correlations of the fluctuations of radar reflectivity and its two moments in cirrus clouds differ depending on the position in the cloud that is, at the top or the bottom or in the bulk of the cloud. Therefore, we study the internal variability of cirrus at isodepths of the cloud. 
- Objective criteria for distinguishing seasons in the western Arctic (Ivanova et al., 2004c) (p. 38)

One characteristic feature of mixed-phase clouds is that, even though they are observed in all seasons at the NSA ARM site, they occur most frequently during the spring and fall transition seasons. The definition and duration of the seasons in the Arctic, however, differs from the standard delineation of the seasons for the mid-latitudes, which is still widely used.

\section{Tracking nucleation-growth-sublimation in cirrus using ARM millimeter wavelength radar observations}

We will discuss in more detail this specific accomplishment because of the breakthroughs it contains and because it is important to demonstrate its potential for future research on characterization of the structure of cirrus properties and its coupling with the state of the large-scale atmosphere and furthermore, on tracing it back to specific formation mechanisms.

\section{Motivation}

We first summarize the motivation behind this research. Cirrus clouds are a crucial component of the earth-atmosphere system because of their upper most position in the atmosphere that defines the essential role which they play in the radiative cloud-climate feedback. Furthermore, the state of the large-scale atmosphere has a profound impact on the formation and maintenance of cirrus clouds. The relationship between the bulk microphysical and radiative properties of cirrus and the synoptic regime, in which they form, however, is still not quantitatively understood.

A fundamental characteristic of cirrus occurrence and maintenance is that there are two different scales of processes. One is the large scale "envelope" of synoptic conditions in which the cirrus is either growing, approximately stationary, or decaying. The other is the small scale of individual generating cells which are always producing new ice crystals. It is obvious that the large scale influences the small scale. It is less obvious that small scale variability influences the large scale. A recent study by Sardeshmukh and Sura (2007) looking at large-scales systems suggests that small-scale variability in diabatic heating does affect the large scale by increasing the amplitude of circulation structures on time scales from the monthly to the interannual. The importance of incorporating small-scale cloud variability and associated diabatic heating into large scale climate and weather models remains an open question.

The interactions between the synoptic conditions and the small scale cloud producing cells are still not quantitatively understood. It is of further interest to possibly trace back small-scale cloud variability to different formation mechanisms. The approach suggested in this study provides a quantitative framework to characterize and assist in understanding of these interactions. 
Knowledge of the local dynamical properties within cirrus and the radiative response due to these properties is still lacking. The coupling between the microphysical, radiative, and dynamical properties of cirrus has been taken into account in modeling studies of cirrus clouds (e.g. [Stephens, 1983]). Early studies by Starr and Cox [1985a, b] using a two-dimensional cirrus cloud model to study the coupling showed that radiative processes strongly regulate dynamical development within a cirrus cloud [Starr and Cox, 1985a,b]. These model results suggest that radar reflectivity observations which express radiative properties of cirrus are appropriate quantity to study in order to improve our understanding of the coupling between the radiative and dynamical properties of cirrus.

In addition to the existence of the two time scale processes, different physical processes are taking place within the depth of the cloud. Aircraft in situ measurements have helped to quantitatively define the structure for typical ice generating cirrus clouds [Heymsfield, 1975, Heymsfield and Miloshevich, 1995]. Aircraft microphysical probes sampled an ice generating cirrus cloud that has up to $20 \%$ ice supersaturation in the upper $2 / 3$ of the cloud and was below ice saturation in the region near cloud base. Under these conditions, ice crystal number concentrations were typically largest in the upper regions of the cloud and smallest near cloud base where aggregation of ice crystals was observed. Using these aircraft measurements, they formulated the structure of cirrus clouds that places an ice generating region near cloud top, an ice crystal growth or deposition region in the middle of the cloud, and sedimentation or sublimation region near the cloud base.

The First International Satellite Cloud Climatology Project Regional Experiment (FIRE) [Cox et al., 1987, Starr, 1987] was among the first to focus on the radiative and microphysical properties of cirrus, but also included studies of the dynamical structure and turbulence within cirrus clouds [Gultepe et al., 1995, Gultepe and Starr, 1995].

The structure of cirrus clouds was part of the study of ice supersaturation in cirrus [Comstock et al., 2004]. Using a unique, year-long dataset of water vapor mixing ratio inferred from ground-based Raman lidar measurements [Comstock et al., 2004] studied the role of ice supersaturation in ice nucleation processes. They found that ice supersaturation occurs $31 \%$ of the time. The large ice supersaturation supports the theory of ice forming homogeneously. They also examined the distribution of ice supersaturation with height and found that in the uppermost portion of a cloud layer, the air is ice supersaturated $43 \%$ of the time which is the ice generating region of the cloud [Heymsfield and Miloshevich, 1995]. Results from our study will be placed into the context of these important experimental studies, field programs and model study results [Khvorostyanov and Sassen, 1998].

The purpose of this research is threefold. First, to further improve the stochastic approach suggested earlier [Ivanova et al., 2006, Ivanova and Ackerman, 2007] for analysis of radar reflectivity observations from cirrus that provides a framework suitable to tackle the two scales of processes which are characteristic of cirrus formation and maintenance. Second, to relate the structure of radiative with the structure of dynamical properties of cirrus and by doing that to improve our understanding of the coupling between radiative and dynamical properties based on remote sensing observations. Third, to place the results into the context of the state of the large-scale atmosphere and to improve our understanding of the structure of dynamical properties at different temporal/spatial 
scales from 1-2 km, to intermediate scales of 6-12 km to larger scales of $100 \mathrm{~km}$.

We aim at deriving a stochastic model for the radiative properties as expressed in the radar reflectivity $\eta(t)$. Because we anticipate that the interactions between the two different scales of processes vary within the depth of cirrus cloud we conduct the analysis of the radar reflectivity at various depth into cloud relative to cloud top. By doing that we present a stochastic model of the two scales of processes at each of the nucleation, growth, and sublimation regions of cirrus.

\section{Observations, PI product, Reanalysis data}

We use data available from the DOE Atmospheric Radiation Measurements (ARM) Program archive for both stochastic analysis and to determine the thermodynamic stratification. We also use NCEP-NARR Reanalysis data to assess the state of the large-scale atmosphere. We will discuss these in more detail below.

We analyze millimeter wavelength cloud radar (MMCR) measurements obtained by the Atmospheric Radiation Measurements (ARM) Program [Ackerman and Stokes, 2003] of the Department of Energy at its Southern Great Plains (SGP) facility near Lamont, OK $\left(36^{\circ} 37^{\prime} \mathrm{N}, 97^{\circ} 30^{\prime} \mathrm{W}\right)$. The ARM MMCR [Moran et al., 1998] is ground-based vertically pointing radar and operates at $35 \mathrm{GHz}$. The radar transmitter emitts an electromagnetic pulse with wavelength $\lambda=8.6 \mathrm{~mm}$, which undergoes elastic scattering from the cloud particles and aerosols. The elapsed time of return of the power scattered back to the radar and the speed of light are subsequently used to infer the distance from the radar to the scattering particles. Hence, the MMCR provides measurements suitable to study the internal structure of clouds.

The average power scattered by the particles to the radar is converted to an estimate of the radar reflectivity $\eta\left(\mathrm{mm}^{2} \mathrm{~m}^{-3}\right)$. The quantity generally used in the radar meteorology field is the radar equivalent reflectivity factor $Z_{e}=\left(\lambda^{4} \eta\right) /\left(\pi^{5} K^{2}\right)$, where $K$ is a physical constant related to the properties of water [Kollias et al., 2007]. Because $Z_{e}$ can span many orders of magnitude, the MMCR data are presented in logarithmic scale $Z=10 \log _{10} Z_{e}$ in units of $\mathrm{dB} Z_{e}$. In this study, however, we analyze the radar equivalent reflectivity factor $Z_{e}$ because the logarithm is a nonlinear operation and we do not want to alter in any way the original data. We will refer to $Z_{e}$ as radar reflectivity $\eta$ because the constant factor $\lambda^{4} /\left(\pi^{5} K^{2}\right)$ does not influence this analysis. Continuous MMCR measurements of $\eta$ are recorded at the SGP facility with $10 \mathrm{~s}$ temporal resolution 24 hours a day and with a $45 \mathrm{~m}$ spatial vertical resolution at 512 height levels. Radar reflectivity data are measured with an accuracy of $0.5 \mathrm{~dB}$.

In this study we examine the internal structure of cirrus cloud observed on April 19, 2004 from 0 to 21 UTC as shown on page 48. Furthermore, because the cirrus properties are undoubtedly a reflection of the history of the air mass in which they form, it is necessary to place the structure of their microphysical and radiative properties into the context of the large-scale dynamics. The history of the air mass indicates that on April 18, 2004 there is a multilayer system - one precipitating cloud and a second, relatively thin cirrus above it. After 23 UTC the two laeyrs colapse into one that continues to exists 
on April 19. This is the cloud that we focus on.

\subsection{Thermodynamic stratification}

Visually the cirrus on April 19 could be considered to contain two parts; one observed from 0 to 7.5 UTC as cirrus part $A$ and a second one from 7.5 to 21 UTC as cirrus part $B$. In order to test such a hypothesis we asses atmospheric thermodynamic structure using ARM data product (SGP-Clouds-Radiation PI Product) derived by merging radiosonde soundings with ground-based data and Rapid Update Cycle (RUC) model output [Mace et al., 2006]. These data represent a characterization of the physical state of the atmospheric column compiled on a five-minute temporal interval and $90 \mathrm{~m}$ vertical grid. Using temperature, pressure, and mixing ratio data we calculate the hourly profiles of the potential $\Theta(K)$ and equivalent potential temperatures $\Theta_{e}(\mathrm{~K})$. Results for $\Theta(\mathrm{K})$ and $\Theta_{e}(\mathrm{~K})$ plotted in Fig. 1 show that there is a neutral thermodynamic stratification from about 9 to $11 \mathrm{~km}$ during cirrus $B$ which did not exist during cirrus $A$. Neutral stratification is usually associated with convection [Gultepe and Starr, 1995]. Having in mind the limitations of the precision of the radiosonde soundings we consider that this result provides a reasonable justification for analyzing the cirrus on April 19 in two parts, $A$ and $B$.

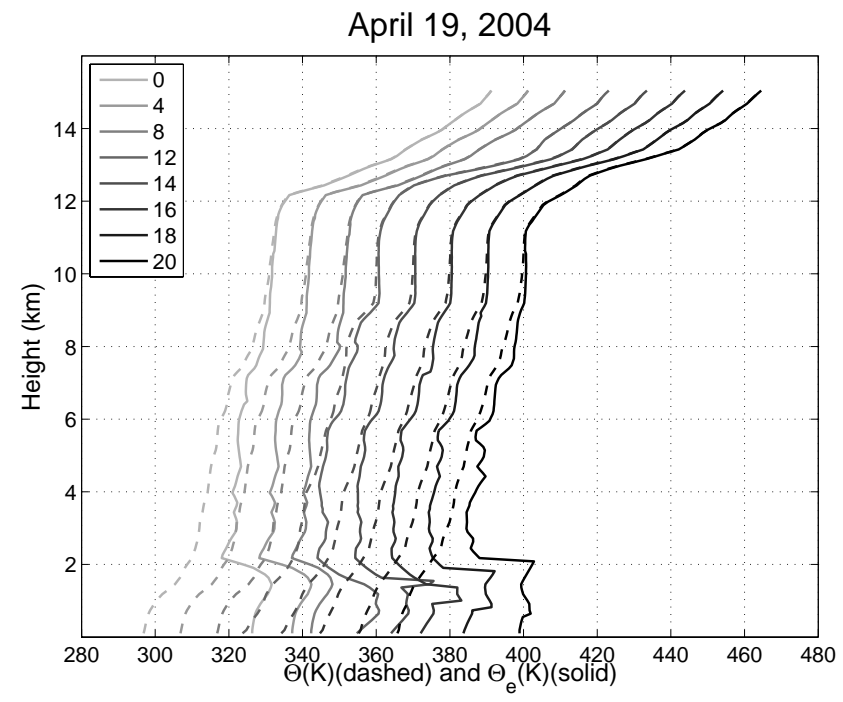

Figure 1: Hourly profiles of equivalent potential temperature $\Theta_{e}(\mathrm{~K})$ (solid curves) and potential temperature $\Theta(\mathrm{K})$ (dashed curves) at $0,4,8,12,14,16,18,20$ UTC on April 19, 2004 derived from ARM data product based on merging radiosonde soundings with ground-based data [Mace et al., 2006]. The most left pair $\Theta_{e}(\mathrm{~K}), \Theta(\mathrm{K})$ curves for 0 UTC are not displaced. Each of the following pairs of $\Theta_{e}(\mathrm{~K}), \Theta(\mathrm{K})$ curves for later times are displaced by $10 \mathrm{~K}$ on the right. Neutral stratification is observed at 12 UTC and after that for heights from about 9 to $11 \mathrm{~km}$. 


\subsection{Temperature assessment}

Recent studies on ice nucleation processes in cirrus clouds based on Raman lidar measurements collected at the ARM SGP site show that large ice supersaturation $(>120 \%)$ is common in cirrus clouds, which supports the theory of ice forming homogeneously [Comstock et al., 2004]. Aircraft in situ measurements show that homogeneous ice nucleation takes place throughout the temperature range from $-35^{\circ}$ to $-56^{\circ} \mathrm{C}$ [Gultepe et al., 1995 , Heymsfield and Miloshevich, 1995]. To assess the ice/water composition of the cloud on April 19, we check the temperature values using again the SGP-Clouds-Radiation PI Product [Mace et al., 2006]. The mean cloud base height being at $7.8 \mathrm{~km}$ has a temperature around $-27^{\circ} \mathrm{C}$. The upper $75 \%$ of the cloud is in the temperature range between $-33^{\circ} \mathrm{C}$ at depth into cloud $d=3 / 4$ and $-55^{\circ} \mathrm{C}$ at the clod top $d=0$. These temperature values suggest that ice crystals in the cloud are forming via homogeneous ice nucleation process.

\subsection{State of the large-scale atmosphere}

To assess the state of the large-scale atmosphere we use the NCEP-NARR, The North American Regional Reanalysis output that has $3 \mathrm{hr}$ time resolution and $32 \mathrm{~km}$ spatial resolution (http://www.meteo.psu.edu/gadomski/NARR/2004.html). It is observed that the low pressure system existing at 21 UTC on April 18 at the surface continues to exist without substantial change through 6 UTC on April 19 at ARM DOE Central facility near Lamont, OK. While the low pressure system moves from southwest to northeast later on that day a high pressure system moves in around 9 UTC. This change in the pressure systems at the surface from low to high is indicative of strong vertical motion due to differential vorticity advection at $500 \mathrm{MB}$ supported by the changes in $500 \mathrm{MB}$ vorticity map (http://www.meteo.psu.edu/ gadomski/NARR/2004.html). Therefore, cirrus $A$ and cirrus $B$ exist in different pressure systems at the surface and different conditions at $500 \mathrm{MB}$ pressure level. We will examine what changes in cirrus structure properties are associated with these different states of the large-scale atmosphere.

\subsection{Defining depths into cloud relative to cloud top}

Because we are interested in investigating the internal structure of cirrus we consider time series of the radar reflectivity $\eta(t)$ at various depths $d=0,1 / 4,1 / 3,1 / 2,2 / 3,3 / 4$, and 1 into the cloud relative to the cloud top as shown in Fig. 2a, and $\mathrm{b}$ for cirrus part $A$ and $B$, respectively.

As an assessment of how the value of the radar reflectivity changes into the depth of the cloud relative to the top we calculate the mean $\langle\eta(t)\rangle$ and the standard deviation $\sigma_{\eta(t)}$ of $\eta(t)$ at each depth level (see Table 1). For both cirrus $A$ and $B$ we obtain an increasing value of both the mean $\left\langle\eta(t)>\right.$ and the standard deviation $\sigma_{\eta(t)}$ from top to $d=3 / 4$ of the cloud depth which is in accordance with what is expected. 

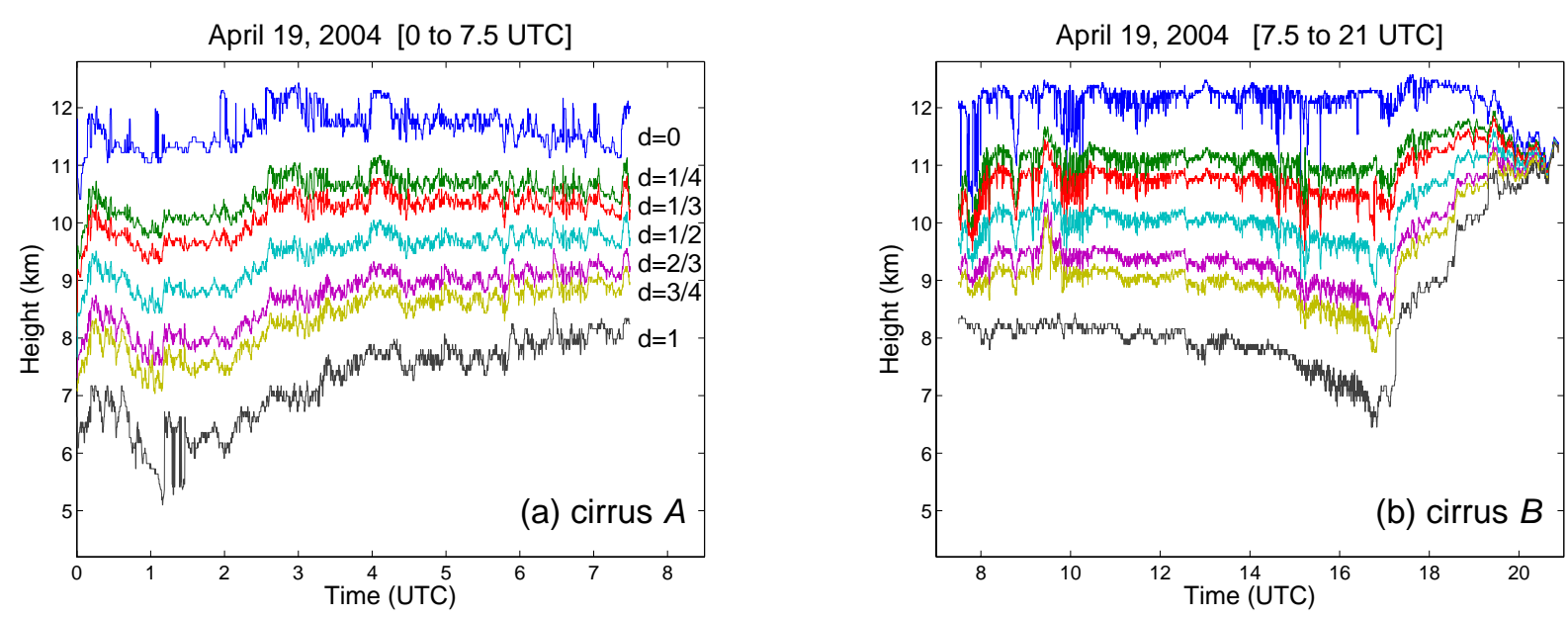

Figure 2: Depth $d$ into cloud relative to cloud top at which time series of the radar reflectivity observations collected with the MMCR on April 19, 2004 are analyzed. (a) cirrus part A. (b) cirrus part $B$.

What we are interested in, however, is what are the rules that govern how the increments of the $\eta(t)$ signal change with time and with depth into the cloud. This will advance our knowledge on cirrus structure and related dynamical processes, as inferred from changes in radiative properties expressed in $\eta(t)$. Furthermore, placing the results into the context of synoptic scale atmosphere has the potential to trace the specific cirrus structure back to specific formation mechanisms.

\section{Probability Distribution Functions}

In order to assess how the increments of the $\eta(t)$ signal change with time and with depth into the cloud we calculate the probability distribution functions ( $p d f s) p(\Delta x, \Delta t$ ) at each depth level for various values of the delay time $\Delta t$. Results for delay time $\Delta t=20 \mathrm{~s}$, $60 \mathrm{~s}, 30 \mathrm{~min}, 1 \mathrm{hr}, 2 \mathrm{hr}$ at various depths $d=0,1 / 4,1 / 3,1 / 2,2 / 3,3 / 4$, and 1 are shown in Fig. 3(a-g) for cirrus $A$ and in Fig. 4(a-g) for cirrus $B$, respectively. We consider normalized increments $\Delta x$ that are calculated as $\Delta x=(\Delta \eta(t)-<\Delta \eta>) / \sigma_{\Delta t}$, where $\Delta \eta(t)=\eta(t+\Delta t)-\eta(t),<>$ denotes the mean and $\sigma_{\Delta t}$ is the standard deviation for the specific delay time. A common feature of these pdfs is that they are strongly nonGaussian, i.e. $p(\Delta x, \Delta t) \neq 0$ for a wide spread of values of the normalized increments $\Delta x= \pm 15$ for almost all values of $\Delta t, d$, and for both $A$ and $B$ cirrus. Several cases, however, make exceptions.

\subsection{Cirrus $A$}

The pdfs of radar reflectivity $\eta$ as shown in Fig. $3(\mathrm{a}-\mathrm{g})$ for time lag $\Delta t=2 \mathrm{hr}$ (tilted triangles) and for various depths $d=0,1 / 4,1 / 3,1 / 2,2 / 3,3 / 4$, and 1 into the cloud can be sorted out in three groups. The first group consists of the pdfs at depth $d=0,1 / 4$, 
and $1 / 3$ (Fig. $3(\mathrm{a}-\mathrm{c})$ ) that exhibit well pronounced positive tails up to $\Delta x= \pm 15$ and lack negative tails beyond Gaussian distribution. The second group consists of the pdfs of $\eta$ at depth $d=2 / 3$, and $3 / 4$ (Fig. 3(e,f)). The tails of these two pdfs are very close to Gaussian distribution not only for time lag $\Delta t=2 \mathrm{hr}$, but also for time lags $\Delta t=30 \mathrm{~min}$, and $1 \mathrm{hr}$. The pdf in Fig. 3(d) for $d=1 / 2$, is a transitional case between group one and group two with a small positive tail. The third group consists of one case only; the pdf at the cloud base $d=1$ (Fig. 3(g)). This pdf exhibits substantial negative tail and small positive tail fatter than the Gaussian distribution. Similar results are obtained for delay time $\Delta t=3 \mathrm{hr}$, but not shown to save space.
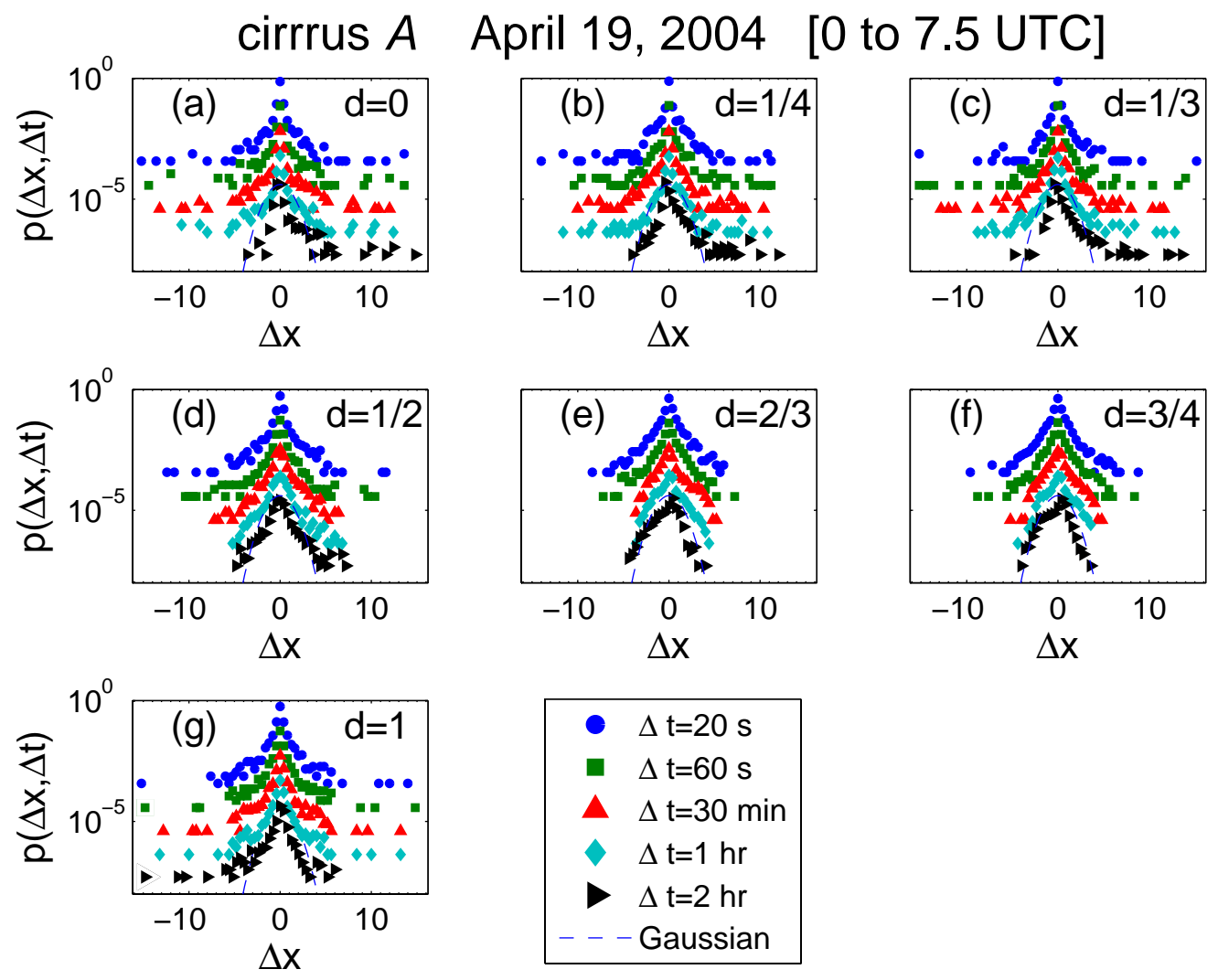

Figure 3: Probability distribution functions $p(\Delta x, \Delta t)$ of radar reflectivity observations $\eta(t)$ for delay times $\Delta t=20 \mathrm{~s}, 60 \mathrm{~s}, 30 \mathrm{~min}, 1 \mathrm{hr}, 2 \mathrm{hr}$ at various depths into cloud relative to cloud top (a) $d=0$; (b) $d=1 / 4$; (c) $d=1 / 3$; (d) $d=1 / 2$; (e) $d=2 / 3$; (f) $d=3 / 4$; (g) $d=1$ for cirrus observed on April 19, 2004 from 0 to 7.5 UTC (cirrus $A$ ). The pdfs are displaced by a factor of 0.1 in vertical for clarity. The pdf for $\Delta t=20 \mathrm{~s}$ is not displaced. Dashed curves mark Gaussian distribution.

These findings indicate that the values of the radar reflectivity $\eta$ in the upper $33 \%$ of cirrus $A$ (Fig. 3(a-c)) become statistically larger after $2 \mathrm{hr}$ most probably owing to the combination of two things. One is the high ice supersaturation which is typical for that region of 
cirrus [Heymsfield and Miloshevich, 1995, Comstock et al., 2004]. The second is the long deposition times needed for deposition of vapor into condensed ice, as found in model studies [Khvorostyanov and Sassen, 1998]. Such a scenario is in agreement with findings by [Comstock et al., 2004] on a cirrus cloud observed at the same (SGP) location on November 15, 2000 and found to have high ice supersaturation region in the middle (in terms of time) of the cloud. This is not typical and in disagreement with the bulk microphysical cirrus and general circulation models which typically assume that ice supersaturation regions quickly deplete excess vapor in 20 - 30 minutes [Khvorostyanov and Sassen, 1998]. At the same time, however, using their explicit cloud model [Khvorostyanov and Sassen, 1998] indicate that deposition of vapor into condensed ice can take several hours, which was reported to be the case on November 15, 2000 and which might be happening on April 19, 2004 between 0 and 7.5 UTC (cirrus $A$ ).

The tails of the pdfs of $\eta$ for time lag $\Delta t=30 \mathrm{~min}, 1,2 \mathrm{hr}$ at depths $d=2 / 3$, and $3 / 4$ (Fig. 3(e,f)) are close to Gaussian distribution which can be interpreted to be related to the system becoming more stable.

The results for the pdf of the radar reflectivity at the cloud base $d=1$ (Fig. 3(g)) indicate that the $\eta$ values become statistically smaller after $2 \mathrm{hr}$ which suggest that most probably sedimentation and/or sublimation took place between $d=3 / 4$ and $d=1$ as expected for this region of cirrus [Heymsfield and Miloshevich, 1995]. These findings are also in agreement with reported lower relative humidity with respect to ice in the lower $25 \%$ of cirrus [Comstock et al., 2004].

\subsection{Cirrus $B$}

Similar to cirrus $A$ the pdfs of $\eta(t)$ for cirrus $B$ are $p(\Delta x, \Delta t) \neq 0$ for a wide spread of values of normalized increments $\Delta x= \pm 15$ for almost all values of $\Delta t$, and $d$ (see Fig. $4(\mathrm{a}-\mathrm{g}))$. An exemption is the pdf of $\eta$ at the top of the cloud $d=0$ (Fig. 4(a)) which for time lag $\Delta t=2 \mathrm{hr}$ is characterized by tails being relatively close to Gaussian distribution. A comparison between the pdfs at the top $(d=0)$ and at the base $(d=1)$ of cirrus $A$ and $B$ shows that these pdfs are different for all values of the delay times $\Delta t$. Such differences in the pdfs can be interpreted to be due to changes in the state of the large-scale atmosphere between cirrus $A$ and $B$ which affect mostly cloud tops and bases. In support to such an explanation is the change in the atmospheric thermodynamic stratification in the upper region from $d=1 / 4$ (around $11 \mathrm{~km}$ ) to $d=0(12.5 \mathrm{~km}$ ) between cirrus $A$ and cirrus $B$ (see Fig. 1).

In summary, the pdfs of observed radar reflectivity $\eta$ at various depth $d$ into the cloud for various delay times $\Delta t$ are non-Gaussian for both cirrus $A$ and $B$. The nonGaussianity of the probability distribution functions can represent many different dynamical systems. Sura et al., (2005) consider for the purpose of illustration two extreme cases: (1) a deterministic model, in which the quantity described by the model is entirely due to a nonlinear deterministic drift perturbed only by a state-independent noise and (2) a stochastic model, in which the quantity is due to a multiplicative noise term with a linear deterministic drift. In the next section we present the method of analysis that we apply to the radar reflectivity observations with the goal of inferring the model that describes 
them.
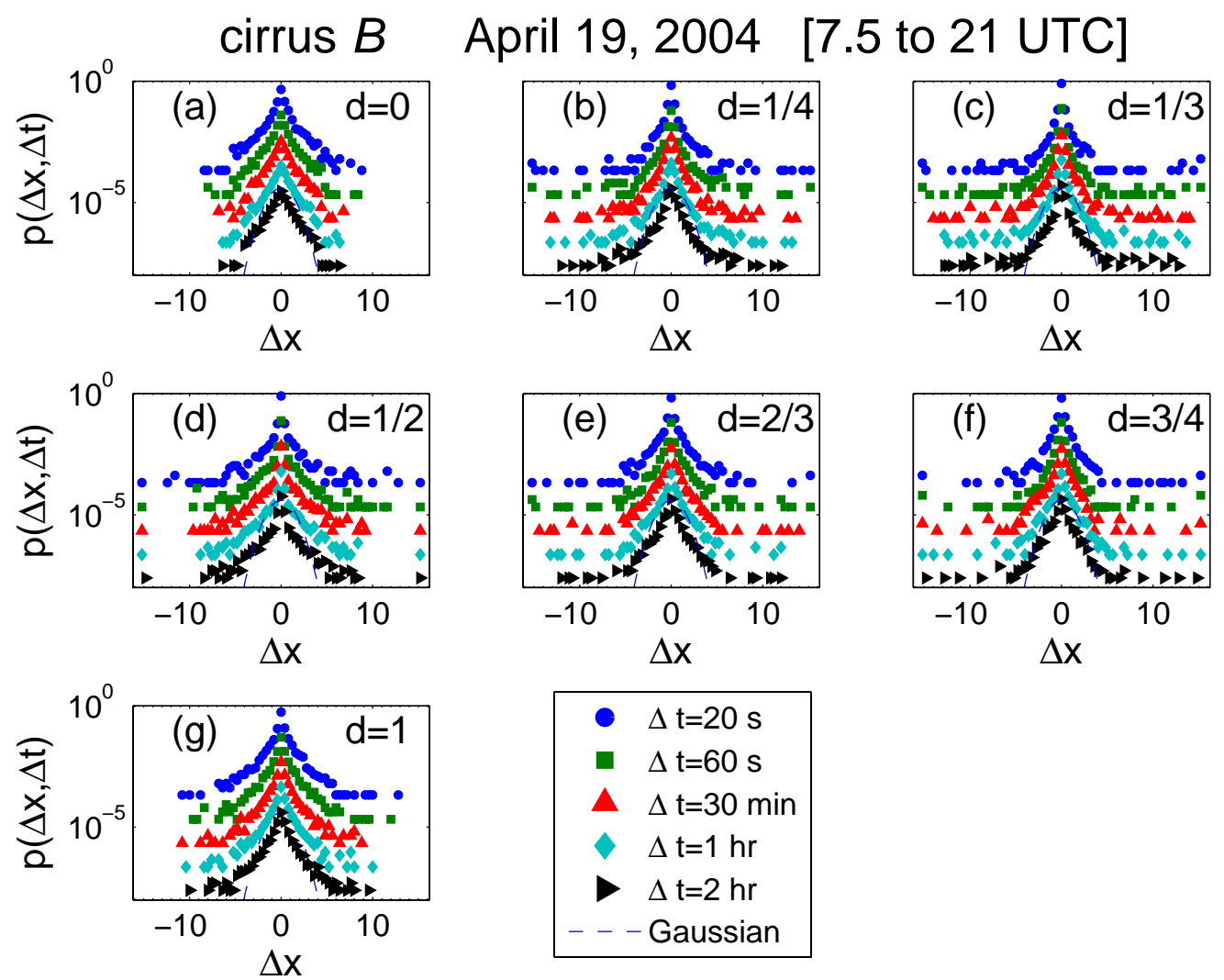

Figure 4: Same as Fig. 3, but for cirrus observed on April 19, 2004 from 7.5 to 21 UTC (cirrus $B)$.

\section{Stochastic Analysis}

The state of the atmosphere is governed by the classical laws of fluid motion, the NavierStokes equations. The problem is that there are no explicit solutions of these equations. Much of our information comes from computer simulations, e.g. [Tetuya et al., 2007], or models based on the cascade idea, e.g. the quantitative model suggested by Kolmogorov and Obukhov [Frisch, 1995] from which among others, scaling exponents of the various moments of the velocity differences can be derived. Alternatively, some progress in treating turbulence can be made by applying methods from statistical mechanics, the Fokker-Planck equation, e.g. to describe turbulence through analysis of the time series of longitudinal component of the velocity recorded during an experiment [Friedrich and Peinke, 1997]. Applying methods of analysis from statistical mechanics allows to take advantage of the ability of statistical mechanics to make macroscopic predictions based on microscopic properties [Friedrich et al., 2000]. 
In this study we apply the Fokker-Planck equation approach to time series of radar reflectivity observations $\eta(t)$ that represent the radiative, microscopic properties at time $t$. In order to quantify the interactions between the two different scales of physical processes in cirrus the Fokker-Planck equation approach involves representing the small-scale rapidly varyng processes as a stochastic forcing [Ivanova et al., 2006, Ivanova and Ackerman, 2007, Sura et al., 2005]. Such stochastic modeling in essence involves approximation of the multiscale dynamical system as depending on the realizations of a white-noise process, that is, as a stochastic differential equation.

In the framework of stochastic dynamics the state vector $\vec{x}$ of an $n$-dimensional system is governed by the stochastic differential equation

$$
\frac{d \vec{x}}{d t}=h(\vec{x}, t)+g(\vec{x}, t) W(t),
$$

called the Langevin equation [Risken, 1984, Gardiner, 2004]. The first term $h(\vec{x}, t)$ is a vector that represents all slow processes in the dynamics of the system. The second term $g(\vec{x}, t) W(t)$ represents the stochastic approximation to the fast nonlinear processes, where in general $g(\vec{x})$ is a matrix and $W(t)$ is the noise vector whose components are independent Gaussian white noise processes. In observational studies because of the limited data record, one can proceed further by considering that the state vector $\vec{x}$ has only two components, e.g. the two leading empirical orthogonal functions of an appropriate atmospheric variable as it is done for example in [Sura et al., 2005, Weisheimer et al., 2001]. Another approach is to consider that Eq. (1) is an equation for the quantity itself expressed by the scalar $x$ as it is done in [Renner et al., 2001, Ivanova et al., 2006, Ivanova and Ackerman, 2007].

In this study we apply the latter to the observations of the radar reflectivity $\eta(t)$. In this case $W(t)$ is $\delta$-correlated Gaussian white noise, $<W(t)>=0,<W(t) W\left(t^{\prime}\right)>=$ $\delta\left(t-t^{\prime}\right)$. The corresponding Fokker-Planck equation describes the conservation of the probability density $p(x, t)$ of the system described by Eq. (1) for $x$ :

$$
\frac{\partial}{\partial t} p(x, t)=\left[-\frac{\partial}{\partial x} D_{1}(x, t)+\frac{\partial^{2}}{\partial x^{2}} D_{2}(x, t)\right] p(x, t),
$$

where the first term is called "drift". For Stratonovich systems this is the term describing the deterministic part of the dynamics of the system. For Itô systems it is interpreted as so-called "effective" drift $D_{1}^{\text {eff.d. }}$. For detailed discussion on Itô and Stratonovich calculi see, for example, [Gardiner, 2004, Penland, 1996]. The type of calculus which must be employed is determined by the physical properties of the system. Stratonovich calculus is relevant for continuous physical systems such as the atmosphere and Itô calculus is used for discrete physical systems. If a stochastic model is indirectly estimated from observed discrete data, however, as it is the case with the present study, then the inferred drift is the effective drift $D_{1}^{\text {eff.d. }}$ and is the sum of the deterministic dynamics (deterministic drift) $D_{1}^{d . d .}$ and the noise-induced drift $D_{1}^{n . i .}$. The second term in Eq. (2) is called "diffusion" because it makes the probability density to defuse. In contrast to time-dependent probability density such as those shown in Fig. 3, the probability density can be stationary and in that case the diffusion is balanced by the drift. 
The drift $D_{1}(x, t)$ coefficient and the diffusion $D_{2}(x, t)$ coefficient are the first two Kramers-Moyal expansion coefficients

$$
D_{n}(x, t)=\left.\frac{1}{n !} \lim _{\Delta t \rightarrow 0} \frac{1}{\Delta t}\left\langle[\xi(t+\Delta t)-x]^{n}\right\rangle\right|_{\xi(t)=x}
$$

where $\xi(t+\Delta t)$ is a solution of Eq. (1) which at time $t$ has the sharp value $\xi(t)=x$. The functional dependence of the drift and diffusion coefficients can be estimated directly from the time series using Eq. (3). Such an approach is employed in [Sura and Barsugli, 2002, Berner, 2005, Berner and Branstator, 2005, Sura et al., 2005].

The functional dependence of the drift and diffusion coefficients can also be estimated directly from the moments $M_{n}(x, t, \Delta t)$ of the conditional probability density

$$
D_{n}(x, t)=\frac{1}{n !} \lim _{\Delta t \rightarrow 0} M_{n}(x, t, \Delta t),
$$

where

$$
M_{n}(x, t, \Delta t)=\frac{1}{\Delta t} \int_{-\infty}^{+\infty}\left(x^{\prime}-x\right)^{n} p\left(x^{\prime}, t-\Delta t \mid x, t\right) d x^{\prime}
$$

for $n=1,2, \ldots$ [Friedrich and Peinke, 1997, Ivanova et al., 2006]. This is the approach used here.

By definition the drift and diffusion coefficients are first and second order moments of the conditional probability distribution functions. The conditional probability distribution functions connect the probabilities at larger scales with the probabilities at all smaller, embedded scales (Eq. (5)). Therefore, owing to their definition (Eqs. $(4,5))$ the two parameters, $D_{1}(x, t)$ and $D_{2}(x, t)$ represent an integral view of the local dynamics.

From Eq. (3) and the integral equation form of Eq. (1) one can obtain the KramersMoyal expansion of the coefficients

$$
\begin{gathered}
D_{1}(x, t)=h(x, t)+\frac{\partial g(x, t)}{\partial x} g(x, t) \\
D_{2}(x, t)=g^{2}(x, t)
\end{gathered}
$$

and $D_{n}(x, t)=0$ for $n \geq 3$ [Risken, 1984]. The drift coefficient which for observed discrete data is the effective drift contains two terms

$$
D_{1}^{e f f . d .}(x, t)=D_{1}^{d . d .}(x, t)+D_{1}^{n . i .}(x, t),
$$

where

$$
D_{1}^{n . i .}(x, t)=g(x, t) \frac{\partial g(x, t)}{\partial x}
$$

is the noise-induced drift and

$$
D_{1}^{d . d .}(x, t)=h(x, t)
$$

is the deterministic drift.

In summary, the Fokker-Planck equation approach provides a method for deriving the equation describing the conservation of the probability distribution function of the 
signal and the equation governing the evolution of the signal starting directly from the observations. In the framework of the FPE the drift coefficient represents the influence of all slow processes in the dynamics of the system and can be tentatively identified with the large-scale deterministic forcing. The diffusion coefficient represents the stochastic approximation to all fast nonlinear processes and can be associated with in-cloud circulation and turbulence.

Therefore, if we find that different deterministic and stochastic forcing is characterizing layers in cirrus with different physics, this would imply that this forcing is amenable to parameterization. Furthermore, because the drift coefficient can be tentatively identified with the large-scale deterministic forcing, this approach has the potential to capture the coupling between the large-scale dynamics and cloud properties. Again, different values in statistical sense would imply that this forcing is amenable to parameterization.

\subsection{Correction Estimate due to Finite-Difference Approxima- tion}

The definitions of drift $D_{1}(x, t)$ and diffusion $D_{2}(x, t)$ coefficients given by Eq. (4) for $n=1$ and 2 are only correct in the limit of $\Delta t \rightarrow 0$. In the case of estimating their values from observed discrete time series, however, the time increment $\Delta t$, even though small, has some finite value. (The time dependence is omitted because we estimate the coefficients at certain time scale $t$.) Therefore, we define finite-difference approximations $\tilde{D}_{1}(x)$ and $\tilde{D}_{2}(x)$ to $D_{1}(x)$ and $D_{2}(x)$, respectively [Friedrich et al., 2002]. Because laws in physics can not depend on the physical units of the quantity, without loss of generality the time interval $\Delta t$ can be rescaled by the discretization step of the time series $\tau=t / \Delta t_{0}$. From the expressions of the conditional moments up to second order of $\tau$ the corrected coefficients are

$$
\begin{gathered}
\tilde{D}_{1}(x)=D_{1}+\frac{\Delta \tau}{2}\left[D_{1} D_{1}^{\prime}+D_{2} D_{1}^{\prime \prime}\right]+\mathcal{O}\left(\Delta \tau^{2}\right) \\
\tilde{D}_{2}(x)=D_{2}+\frac{\Delta \tau}{2}\left[D_{1}^{2}+2 D_{2} D_{1}^{\prime}+D_{1} D_{2}^{\prime}+D_{2} D_{2}^{\prime \prime}\right]+\mathcal{O}\left(\Delta \tau^{2}\right),
\end{gathered}
$$

where $/$ denotes derivative with respect to $x$.

Note that corrections to the drift and diffusion coefficients can also be obtained in the framework of their derivation directly from the time series as this is suggested by [Sura and Barsugli, 2002]. The corrections to the two coefficients due to the finitedifference approximation are given by the differences $\tilde{D}_{1}-D_{1}$ for the drift and $\tilde{D}_{2}-D_{2}$ for the diffusion coefficients.

\section{Results and Discussion}

We apply the Fokker-Planck equation approach to obtain the functional dependence of the drift coefficient $D_{1}(x)$ and the diffusion coefficient $D_{2}(x)$ of the Fokker-Planck equation Eq. (2) for each of the time series of radar reflectivity $\eta(t)$ at various depths $d=0,1 / 4$, 
$1 / 3,1 / 2,2 / 3,3 / 4$, and 1 into the cloud relative to cloud top and for both cirrus $A$ and $B$. To illustrate the method we choose the time series of radar reflectivity at $d=1 / 2$ in cirrus $B$. Results for the drift coefficient $D_{1}(x)$ and the diffusion coefficient $D_{2}(x)$ are obtained directly from the observational data using Eqs. (4), and (5) for scale $\tau=3 \tau_{\min }$ $\left(\tau_{\min }=2 \Delta t_{0}\right)$ and scale difference $\Delta \tau=2 \tau_{\min }$ (see Fig. $5 \mathrm{a}$, and b).
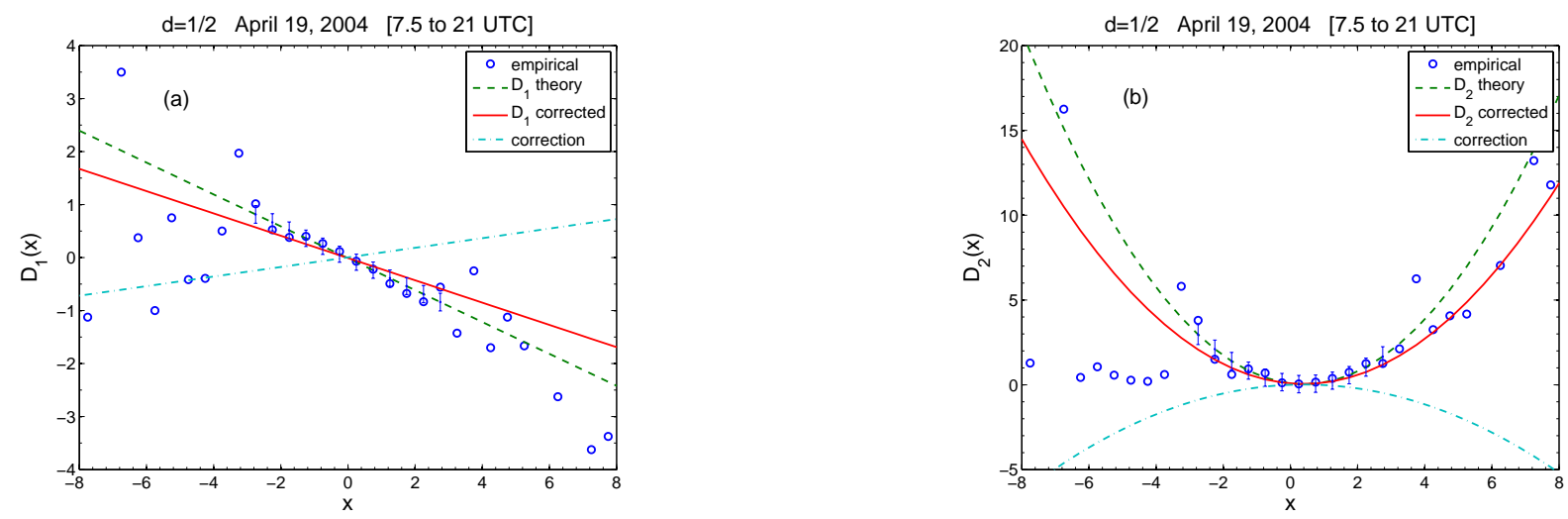

Figure 5: Functional dependence of (a) the effective drift and (b) the diffusion coefficient at scale $\tau=3 \tau_{\min }$ and scale difference $\Delta \tau=2 \tau_{\min }$ of the normalized increments of the radar reflectivity $\eta(t)$ at depth into cloud $d=1 / 2$ relative to cloud top for cirrus $B$. Symbols present the inferred effective drift $D_{1}(x)$ and diffusion $D_{2}(x)$ coefficients from observations Eq. (4). Dashed lines and curves mark the least-square fit to $D_{1}(x)$ and $D_{2}(x)$. Marked is the standard error of the least-square fitting and the fitting interval of $x$-values. The values of the correlation coefficient $R_{c}$ of the least-square fitting to obtain the linear dependence of $D_{1}(x)$ and the values of the confidence interval for the leading linear coefficient are listed in Table 1. Solid lines and curves denote $\tilde{D}_{1}(x)$ and $\tilde{D}_{2}(x)$, which are the drift and diffusion coefficients corrected for using the finite-difference approximation Eqs. (11) and (12). Dot-dashed lines and curves mark the corrections to drift and diffusion, $\tilde{D}_{1}(x)-D_{1}(x)$ and $\tilde{D}_{2}(x)-D_{2}(x)$, respectively.

Because we obtain the drift from observed discrete data, it is the effective drift $D_{1}^{e f f . d .}(x)$ that is plotted with symbols (circles) in Fig. 5a. Owing to the linear dependence of the drift coefficient with $x$ the second term in the brackets in Eq. (11) is equal to zero, e.g. $D_{1}^{\prime \prime}=0$. Therefore, the correction to the drift is linear as marked by the dot-dashed line in Fig. 5a. After correcting for using finite-difference approximation by applying Eq. (11) we find that the corrected effective drift plotted with solid line $\tilde{D}_{1}^{\text {eff.d. }}$ shows a linear dependence with $x$, e.g. $\tilde{D}_{1}^{e f f . d .}=a_{1} x+a_{0}$, where $a_{1}=-0.210 \pm 0.025$ and $a_{0}=0.009$. The values of the correlation coefficient $R$ of the least-square fitting to obtain the linear dependence of $D_{1}(x)$ and the values of the confidence interval $\epsilon$ for the leading linear coefficient $a_{1}$ are listed in Table 1 .

The diffusion coefficient $D_{2}(x)$ calculated from Eqs. (4) and (5) for $n=2$ is plotted with symbols in Fig. 5b together with its least-square fit (dashed line). We obtain that the 
Table 1: Statistical parameters of the radar reflectivity $\eta$ at various depths $d$ into cloud relative to cloud top for the cirrus $A$ and $B$ observed on April 19, 2004. $N$ marks the number of the observational data in each time series of $\eta .\langle\eta\rangle$ and $\sigma_{\eta}$ denote the mean and standard deviation of $\eta$ for the time period specified. $R$ is the correlation coefficient of the least-square fitting to obtain the linear dependence of the drift coefficient $\tilde{D}_{1}{ }^{\text {eff.d. }}(x)=a_{1} x+a_{0}$ and $\epsilon$ is the confidence interval of the leading linear coefficient $a_{1}$. The leading coefficient $b_{2}$ of the second order polynomial dependence of the corrected diffusion coefficient $\tilde{D}_{2}(x)=b_{2} x^{2}+b_{1} x+b_{0}$ is listed in column six. The last column contains values of the pdfs for time lag $\Delta t=3 \tau_{\min }=60 \mathrm{~s}$ at $\Delta x=0$.

\begin{tabular}{|c|c|c|c|c|c|c|}
\hline $\bar{d}$ & $<\eta>$ & $\sigma_{\eta}$ & $R$ & $a_{1} \pm \epsilon$ & $b_{2}$ & $p(\Delta x=0)$ \\
\hline \multicolumn{2}{|c|}{ Cirrus $A$} & \multicolumn{2}{|c|}{ April 19, 2004} & \multicolumn{2}{|c|}{ [0 to 7.5 UTC] } & $N=2697$ \\
\hline 0 & 0.0011 & 0.0026 & 0.90 & $-0.221 \pm 0.038$ & 0.105 & 0.710 \\
\hline $1 / 4$ & 0.0366 & 0.0771 & 0.96 & $-0.190 \pm 0.022$ & 0.075 & 0.746 \\
\hline $1 / 3$ & 0.0647 & 0.1327 & 0.93 & $-0.201 \pm 0.027$ & 0.173 & 0.740 \\
\hline $1 / 2$ & 0.1699 & 0.2018 & 0.99 & $-0.200 \pm 0.014$ & 0.293 & 0.535 \\
\hline $2 / 3$ & 0.4328 & 0.4609 & 0.98 & $-0.214 \pm 0.016$ & 0.143 & 0.408 \\
\hline $3 / 4$ & 0.5657 & 0.5676 & 0.99 & $-0.189 \pm 0.007$ & 0.088 & 0.425 \\
\hline 1 & 0.0059 & 0.0119 & 0.98 & $-0.229 \pm 0.022$ & 0.114 & 0.551 \\
\hline \multicolumn{2}{|c|}{ Cirrus $B$} & \multicolumn{2}{|c|}{ April 19, 2004} & $4 \quad[7.5$ to 21 & UTC] & $N=4760$ \\
\hline$\overline{0}$ & 0.0002 & 0.0003 & 0.97 & $-0.203 \pm 0.014$ & 0.099 & 0.402 \\
\hline $1 / 4$ & 0.0023 & 0.0030 & 0.93 & $-0.220 \pm 0.027$ & 0.146 & 0.612 \\
\hline $1 / 3$ & 0.0045 & 0.0092 & 0.96 & $-0.233 \pm 0.027$ & 0.127 & 0.755 \\
\hline $1 / 2$ & 0.0193 & 0.0431 & 0.94 & $-0.210 \pm 0.025$ & 0.205 & 0.754 \\
\hline $2 / 3$ & 0.0420 & 0.0732 & 0.90 & $-0.204 \pm 0.033$ & 0.171 & 0.636 \\
\hline $3 / 4$ & 0.0601 & 0.1033 & 0.95 & $-0.216 \pm 0.024$ & 0.220 & 0.642 \\
\hline 1 & 0.0013 & 0.0022 & 0.93 & $-0.232 \pm 0.034$ & 0.134 & 0.503 \\
\hline
\end{tabular}


diffusion coefficient is not a constant, i.e. $D_{2}(x)$ is state-dependent. Therefore, the fast processes in the dynamics of the system are described by state-dependent, multiplicative noise. Marked also are the standard error of the fit as well as the fitting interval of $x$ values. The correction for using finite-difference approximation is calculated from Eq. (12) and plotted with dot-dashed line. It is found that the corrected diffusion coefficient $\tilde{D}_{2}(x)$ can be approximated by a polynomial of degree two $\tilde{D}_{2}(x)=b_{2} x^{2}+b_{1} x+b_{0}$, where $b_{2}=0.205, b_{1}=-0.16$ and $b_{0}=0.08$ (see Fig. 5b and Table 1 ).

The values of the correlation coefficient $R$ of the linear least-square fit of $D_{1}(x)$, the values of the leading linear coefficient $a_{1}$, its confidence interval $\epsilon$, and the leading quadratic coefficient $b_{2}$ of the diffusion coefficient for all time series of radar reflectivity at various depth $d$, are summarized in Table 1 and plotted in Fig. 6a, and b for cirrus $A$ and $B$, respectively.
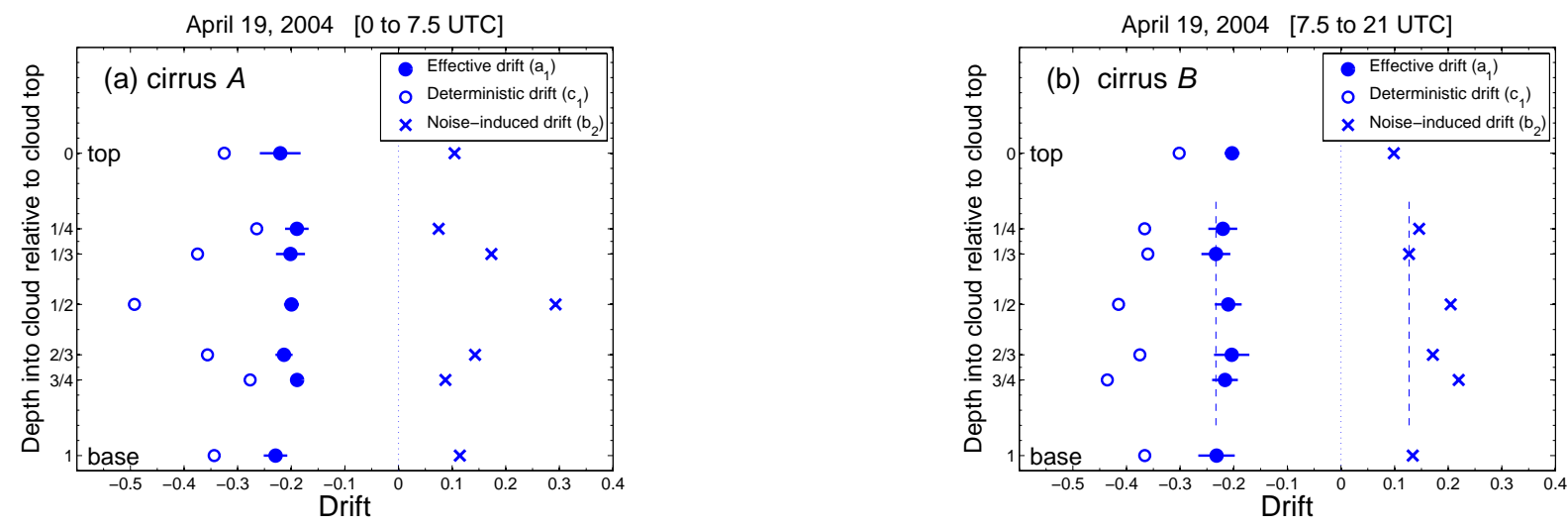

Figure 6: Values of the leading linear coefficients describing the effective, deterministic and noise-induced drift of the radar reflectivity $\eta$ at various depths $d$ into the cloud relative to cloud top $d=0,1 / 4,1 / 3,1 / 2,2 / 3,3 / 4$, and 1 for cirrus observed on April 19 (a) from 0 to 7.5 UTC (cirrus $A$ ) and (b) from 7.5 to 21 UTC (cirrus $B$ ). Bold dots present the leading linear coefficient $a_{1}$ of the inferred effective drift $\tilde{D}_{1}^{\text {eff.d. }}(x)=a_{1} x+a_{0}$. Cross signs denote the leading linear coefficient $b_{2}$ of the noise-induced drift $\tilde{D}_{1}^{n . i .}(x)=b_{2} x+b_{1} / 2$ (Eq. (9)) owing to the statedependent multiplicative noise, e.g. nonconstant diffusion. Circles mark values of the linear coefficient $c_{1}$ of derived deterministic drift $\tilde{D}_{1}^{d . d .}(x)=c_{1} x+c_{0}$ (Eq. (10)). The short horizontal lines across the bold dots present the confidence interval of the leading linear coefficient obtained from the linear least-square fit (see Table 1 and Fig. 5a). (b) Dashed vertical lines mark the largest value of $\left|a_{1}\right|$ and the smallest value of the noise-induced drift $b_{2}$ for radar reflectivity time series in the bulk of the cloud, e.g. outside the top and the base of cirrus $B$.

The diffusion coefficient $\tilde{D}_{2}(x)$ contributes to the drift via noise-induced drift Eq. (9). It follows from Eqs. (7) and (9) that the quadratic dependence of the diffusion coefficient $\tilde{D}_{2}(x)=b_{2} x^{2}+b_{1} x+b_{0}$ results in linear noise-induced drift $\tilde{D}_{1}^{n . i}(x)=b_{2} x+b_{1} / 2$. The values of the leading coefficient $b_{2}$ of the noise-induced drift are plotted with crosses in Fig. 6a,b and mark the noise-induced drift $\tilde{D}_{1}^{n . i .}(x)$. Using Eq. (8) one can obtain the 
deterministic drift $\tilde{D}_{1}^{\text {d.d. }}(x)=c_{1} x+c_{0}$ in the dynamics of the system. The deterministic drift is represented by the values of $c_{1}$ in Fig. 6a,b (circles).

Next we need to make some syntax of the effective drift presented by the values of $a_{1}$, and the noise-induced drift or diffusion presented by the values of $b_{2}$ as shown in Fig. 6a, and b. For cirrus $A$ the values of $\left|a_{1}\right|$ for the pdf of $\eta$ time series at the top and at the base of the cloud are larger in comparison to the $\left|a_{1}\right|$ values for the bulk of the cloud (see Fig. 6a). This can be understood in view of the meaning of the drift which in essence is some mean tendency. The top and the base of the cloud are directly influenced by the large-scale dynamics. Therefore, the tendency is expected to be most pronounced at the top and the base versus the bulk of the cloud, especially when there are strong features in the large-scale dynamics. This influence is expressed in the larger values of $\left|a_{1}\right|$. The fact that different characteristics are obtained for the top and the base versus the bulk of the cloud is in accordance with results from previous studies [Ivanova et al., 2003, Ivanova et al., 2006].

For both cirrus $A$ and $B$ the least-square fit of the diffusion coefficient $D_{2}(x)$ is characterized by larger error versus the error for the linear fit of the drift coefficient $D_{1}(x)$. With that in mind we find that both the upper and lower $25 \%$ of cirrus $A$ (Fig. 6a) and both upper $33 \%$ and lower $<25 \%$ of cirrus $B$ (Fig. $6 \mathrm{~b}$ ) are characterized by values of the noise-induced drift $b_{2}$, that are relatively small in comparison to the middle $50 \%$ of the cloud.

The noise-induced drift represents the stochasticity in the dynamics of the system as expressed by the radar reflectivity. Even though the upper $25 \%$ of cirrus is the ice generating region of the cloud [Heymsfield and Miloshevich, 1995] there is no much diffusivity in the pdf of $\eta(t)$ because the ice crystals are still small. The radar reflectivity being proportional to the sixth power of the effective radius of the particles is not characterized by big fluctuations due in part to the small ice crystal effective radius. See also Table 1 for the values of the mean and standard deviation of $\eta(t)$. The lower $25 \%$ of cirrus is the sublimation or sedimentation region of the cloud. The effective radius of the ice crystals in this region is large but because the growth and deposition process is already completed, there is no much spread in the distribution of particle sizes and therefore there is no much spread in the pdf of radar reflectivity, no much diffusivity.

The middle portion of cirrus is the ice crystal growth and deposition region [Comstock et al., 2004], [Heymsfield and Miloshevich, 1995]. We find that in this region the values of $b_{2}$ are larger at depth into the cloud $d=1 / 3,1 / 2$, and $2 / 3$ for cirrus $A$ (see Fig. 6 a) and at $d=1 / 2$, $2 / 3$, and $3 / 4$ for cirrus $B$ (see Fig. $6 \mathrm{~b}$ ). These larger values of $b_{2}$ which mean larger diffusivity of the pdfs are in accordance with what is expected from the ice crystal growth and deposition region. The analysis of the functional dependence of the drift and diffusion coefficients obtained from the moments of conditional probability distribution (Eq. (4)) is performed at time scale of $\tau=3 \tau_{\min }=60 \mathrm{~s}$. For wind speed of $10-20 \mathrm{~m} / \mathrm{s}$ this time scale translates into spatial scales of $0.6-1.2 \mathrm{~km}$ which are typical scales for the cirrus generating cells [Heymsfield, 1975, Starr and Quante, 2002]. One of the principal mechanisms of cirrus cloud maintenance is through generating cells. The existence of these cells was predicted by model results [Starr and Cox, 1985a, Starr and Cox, 1985b]. Because of their fine structure $(<1 \mathrm{~km})$, they were identified later by active remote sensing during the 
FIRE field program [Sassen et al., 1990, Sassen et al., 1995] and by in-situ measurements [Quante and Brown, 1992, Gultepe and Starr, 1995, Gultepe et al., 1995]. Therefore, the stochastic approach applied here allows for analyzing the cirrus at its finest scales, but also to distinguish between the cooperative results from all slow processes and the fast non-linear processes that determine the dynamics of the system. It is very likely that in the middle region of cirrus during the course of ice crystal growth and deposition there is a high variability in particle sizes, ice crystal concentrations, and in radar reflectivity that are expressed in the larger diffusivity $\left(b_{2}\right)$ obtained for these pdfs. (See also Table 1, and 2).

These findings support the presumption that the multiplicative noise, e.g. the noiseinduced drift can be associated with in-cloud circulation and turbulence. They also indicate that cirrus $A$ is predominately in the state of growing, while cirrus $B$ is in the state of decaying.

Note that the layer at depth of cloud $d=3 / 4$ in cirrus $B$ that is characterized with neutral stratification has values of the leading drift coefficient $a_{1}$ and diffusion coefficient $b_{2}$ that satisfy the relation $a_{1}=2 b_{2}$ (see Table 1 ). As we previously reported, this relation holds true for fully developed turbulence according to the $4 / 5$ Kolmogorov law (Ivanova et al., 2006, Ivanova and Ackerman, 2007c).

Another argument in analyzing the results for the drift and the diffusion coefficients is of geometric nature and it comes from the manner in which $D_{1}$ and $D_{2}$ are related to the shape of the pdf. In principal, the larger the effective drift $\left|a_{1}\right|$ the higher the pdf peak and the larger the noise-induced drift (diffusion) $b_{2}$, the fatter the pdf [Penland, 2003]. This is expected to be obtained for the ideal case of infinitely long time series of observations. For the real observations we aim at understanding the results in view of this concept but we also keep in mind that the limited number of observations may blur these relationships.

The radar reflectivity time series of cirrus $B$ are of length $N=4760$ data points which is longer than $N=2697$ for cirrus $A$ (see Table 1 ). This might be one of the reasons for a better correspondence between the values of the pair $\left(\left|a_{1}\right|, b_{2}\right)$ (Table 1$)$ for $\eta(t)$ time series in the bulk of cirrus $B$ and the shape of the pdfs for delay time $\Delta t=3 \tau_{\min }$ (see Fig. 7 and Table 1 for the values of $p(\Delta x=0)$ ).

Dashed lines in Fig. 6b are drawn to pass through the maximum value of the effective drift coefficient $\left|a_{1}\right|$ and through the minimum value of the noise-induced drift coefficient $b_{2}$. It appears that the pair $\left(\max \left(\left|a_{1}\right|\right), \min \left(b_{2}\right)\right)$ characterizes the dynamics of $\eta(t)$ at depth $d=1 / 3$. If the concept described above was to hold true, this means that the pdf for $\eta(t)$ at $d=1 / 3$ should have simultaneously the highest peak and the most narrow shoulders among the pdfs for the other depths and this is exactly what is observed in Fig. 7 (diamonds) (see also Table 1). The rest of the pdfs have wider shoulders (Fig. 7) that correspond to larger values of $b_{2}$, e.g. on the right of (right) dashed line in Fig. 6b. They also have lower peaks that correspond to smaller values of $\left|a_{1}\right|$, e.g. on the right of (left) dashed line in Fig. 6b. 


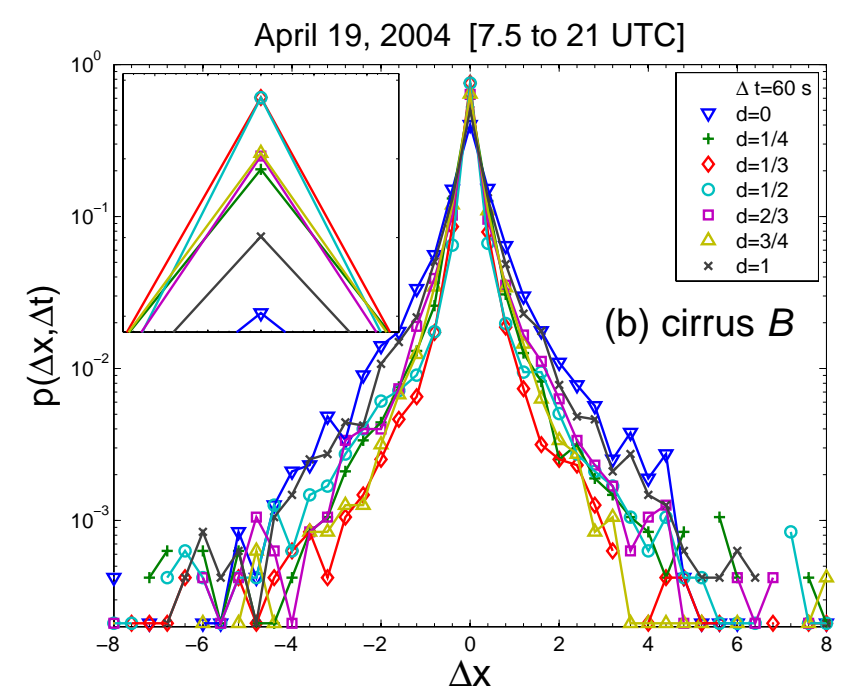

Figure 7: Probability distribution functions $p(\Delta x, \Delta t)$ of radar reflectivity observations $\eta(t)$ for delay time $\Delta t=60 \mathrm{~s}$ at various depths into cloud relative to cloud top $d=0$ (upside triangle), $d=1 / 4$ (plus), $d=1 / 3$ (diamond), $d=1 / 2$ (circle), $d=2 / 3$ (square), $d=3 / 4$ (triangle), and $d=1$ (cross) for cirrus $B$. The inset is a blow up of the peaks of the pdfs.

\subsection{How Justifiable is the White Noise Approximation}

In order to place more value in understanding of these results we need to assess how justifiable is the stochastic modeling of the radar reflectivity. It is important to assess if the white noise approximation made in Eq. (1) is justifiable for the radar reflectivity observations used. Using the moments of the conditional probability distribution Eq. (4) we obtained the effective drift $\tilde{D}_{1}^{\text {eff.d. }}(x)$ directly from the observations. Ideally, the difference between the observation and derived value at each time $t+\Delta t$,

$$
r=x_{o b s}(t+\Delta t)-x(t+\Delta t)
$$

should be equal to zero if the white-noise assumption is correct. Using the Langevin equation Eq. (1) in finite-differences and estimated effective drift $\tilde{D}_{1}^{\text {eff.d. }}(x)$ for each observation we can calculate

$$
x(t+\Delta t)=x_{o b s}(t)+\tilde{D}_{1}^{e f f \cdot d \cdot}(x) \Delta t+C,
$$

where $C=W(t) \sqrt{D_{2}(x)} \Delta t$ is the white-noise term. Replacing the expression for $x(t+\Delta t)$ from Eq. (14) in Eq. (13) leads to

$$
r=\Delta x_{o b s}-\left(\tilde{D}_{1}^{e f f . d .}(x) \Delta t+C\right) .
$$

In statistical sense $r$ will be equal to zero, if the residual $r_{0}$

$$
r_{0}=\Delta x_{o b s}-\tilde{D}_{1}^{e f f . d .}(x) \Delta t
$$


is equal to $C$ in statistical sense, e.g. $r_{0}$ should behave statistically as white noise. In order to test the hypothesis that $r_{0}$ behaves as white noise, we calculate the autocorrelation function of the residual term $r_{0}$ for radar reflectivity at various depth $d=0,1 / 4,1 / 3,1 / 2$, $2 / 3,3 / 4$, and 1 , using the linear dependence for $\tilde{D}_{1}^{\text {eff.d. }}(x)=a_{1} x+a_{0}$ and values for $a_{1}$ and $a_{0}$ which are listed in Table 1 . The results for the autocorrelation function of the residual term $r_{0}$ are shown in Fig. 8a, and b for cirrus $A$ and cirrus $B$, respectively.
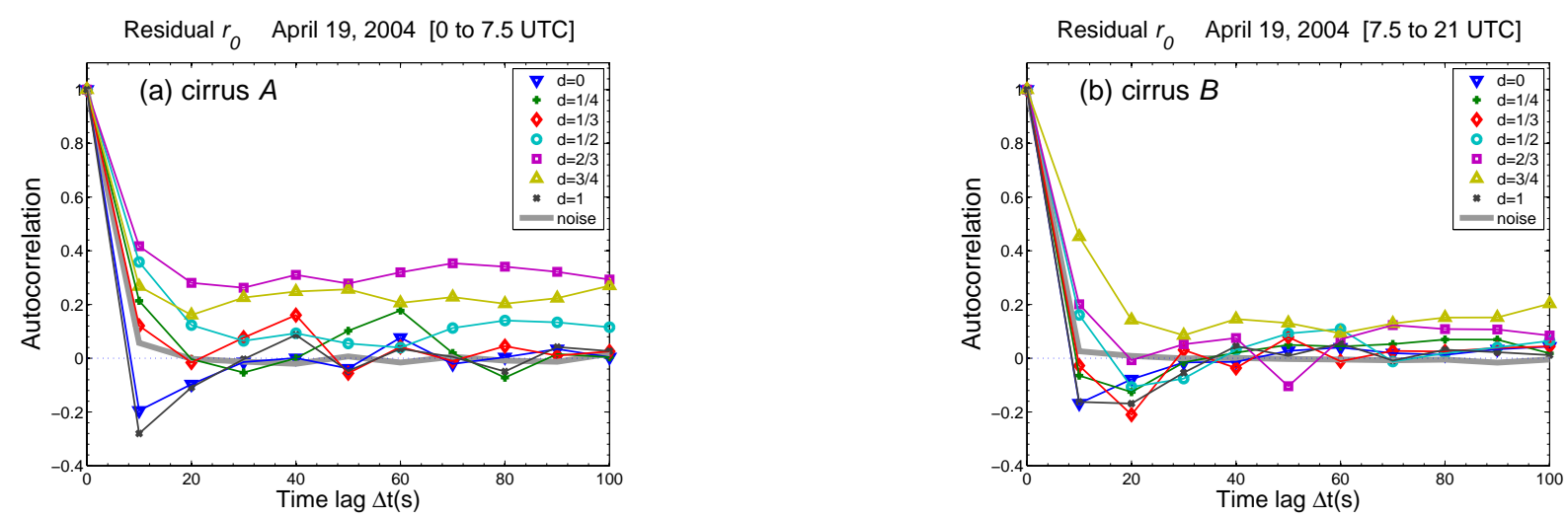

Figure 8: Autocorrelation functions of the residuals $r_{0}$ at various depths into cloud relative to cloud top $d=0$ (upside triangle), $d=1 / 4$ (plus), $d=1 / 3$ (diamond), $d=1 / 2$ (circle), $d=2 / 3$ (square), $d=3 / 4$ (triangle), and $d=1$ (cross) for (a) cirrus $A$ and (b) cirrus $B$. The thick gray curve marks the autocorrelation function of normally distributed Gaussian white noise.

The autocorrelation functions for most of the residuals for cirrus $B$ (Fig. 8b) are uncorrelated at the resolved time scale, i.e. time lag $\Delta t=20 \mathrm{~s}$ which is equal to $\tau_{\min }$ and therefore less than the scale $3 \tau_{\min }=60 \mathrm{~s}$ at which the drift and diffusion coefficients are obtained from the moments of the conditional probability Eq. (4). The autocorrelation function for the residual at depth $d=3 / 4$ (triangles in Fig. 8b) does not cross the zero line (dotted) although its value is close to zero $(\approx 0.09)$ for time lag $\Delta t=30 \mathrm{~s}$, which is less than $3 \tau_{\min }$. Therefore, the white-noise assumption is justifiable for the radar reflectivity time series in cirrus $B$.

The results for the autocorrelation function of the residual term $r_{0}$ for cirrus $A$ plotted in Fig. 8a show that the autocorrelation function is uncorrelated for all depths except for $d=2 / 3$ (squares), and $3 / 4$ (triangles). The autocorrelation function for the residual at depth $d=1 / 2$ does not cross the zero line (dotted) but its value is very close to zero $(\approx 0.04)$ for time lag $\Delta t=60 \mathrm{~s}$. Therefore, we may consider that the white-noise assumption is justifiable for the radar reflectivity time series in cirrus $A$ for all depths except for $d=2 / 3$, and $3 / 4$. Because of that we should be cautious when interpreting the results obtained for the diffusion coefficient $D_{2}(x)$ at depths $d=2 / 3$, and $3 / 4$ into cloud relative to cloud top for cirrus $A$. 


\subsection{Correlations and Periodicity}

To study further the correlations in radar reflectivity $\eta(t)$ time series in cirrus $A$ and compare them with the correlations in cirrus $B$ we calculate the autocorrelation function of $\eta(t)$ time series. The results for depth in cloud $d=0,1 / 4$, and $1 / 3$ are shown in Fig. $9 \mathrm{a}(\mathrm{c})$ for cirrus $A(B)$ and the results for depth in cloud $d=1 / 2,2 / 3$, and $3 / 4$, and 1 are shown in Fig. $9 \mathrm{~b}(\mathrm{~d})$ for cirrus $A(B)$. There is a clear difference between the ACFs of cirrus $A$ and cirrus $B$. The existence of relatively high correlations in $\eta(t)$ time series at depths $d=2 / 3$, and $3 / 4$ in cirrus $A$ (see Fig. 9c) for a broad range of values of the time lag makes it difficult to find the time scale that separates the fast from the slow processes. This appear to be the reason for the correlations in the autocorrelation functions of the respective residuals $r_{0}$ discussed in previous subsection. At the same time, we need to point out that it is very difficult to separate the fast from the slow time scale in the atmosphere [Sura et al., 2005].
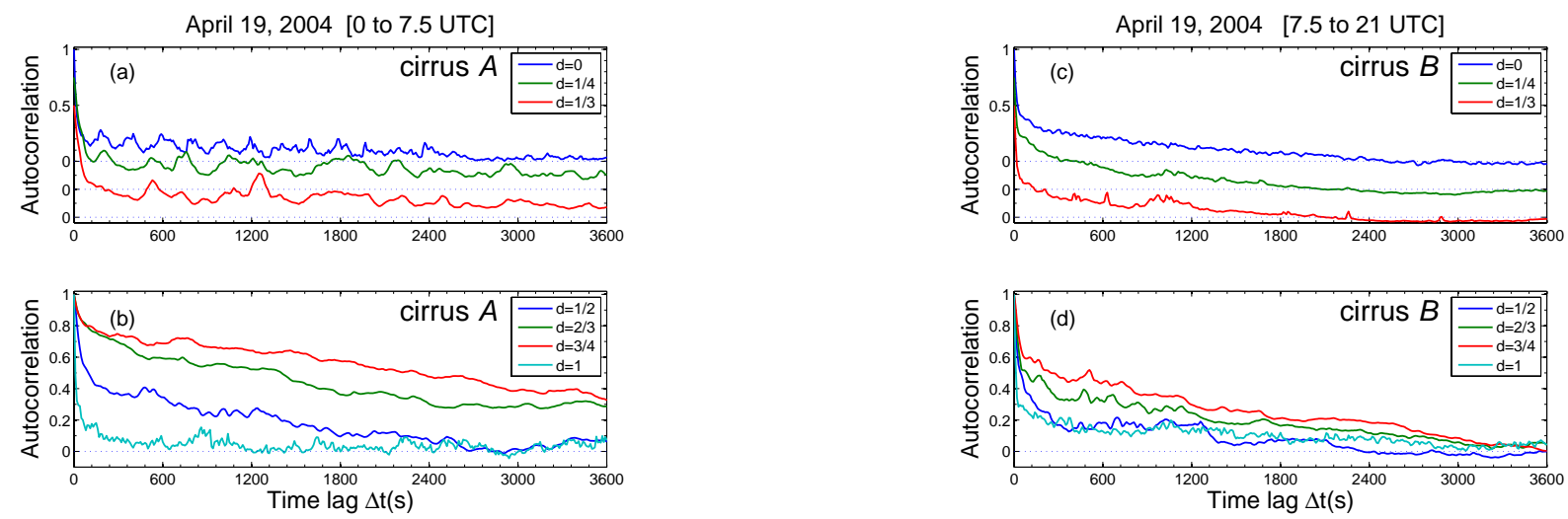

Figure 9: Autocorrelation functions $(\mathrm{ACF})$ of radar reflectivity $\eta(t)$ at various depths relative to cloud top into cirrus cloud (a,b) $A$ and (c,d) $B$. (a,c) from top to bottom $d=0$ (blue), $d=1 / 4$ (green), $d=1 / 3$ (red). Each ACF is displaced downward by -0.25 relative to the previous and dotted lines mark the zero ACF value. (b,d) from top to bottom $d=3 / 4$ (red), $d=2 / 3$ (green), $d=1 / 2$ (blue), and $d=1$ (cyan).

The autocorrelation functions of $\eta(t)$ time series at depth $d=0,1 / 4,1 / 3$ in cirrus $A$ (see Fig. 9a) clearly exhibit periodicities. To find the length of the periods we use fast Fourier transform of $\eta(t)$ time series. We find that the time series of radar reflectivity at the top of the cloud $d=0$ fluctuate with a period of $w_{1}=200 \mathrm{~s}$ as shown in Fig. 10. The time series of $\eta(t)$ at both depths $d=1 / 4$, and $1 / 3$ fluctuate with a period of $w_{2}=600 \mathrm{~s}$. We also tested for periodicities the $\eta(t)$ time series at the rest of the depths of cloud $A$. At depth $d=3 / 4$ in cirrus $A$ we find two peaks in the power with close values of the amplitudes. The higher peak is at $w_{3}=820 \mathrm{~s}$ and the lower is at $w_{4}=700 \mathrm{~s}$. that radar reflectivity fluctuates with period of No periodicities are found at any depth in cirrus $B$. 

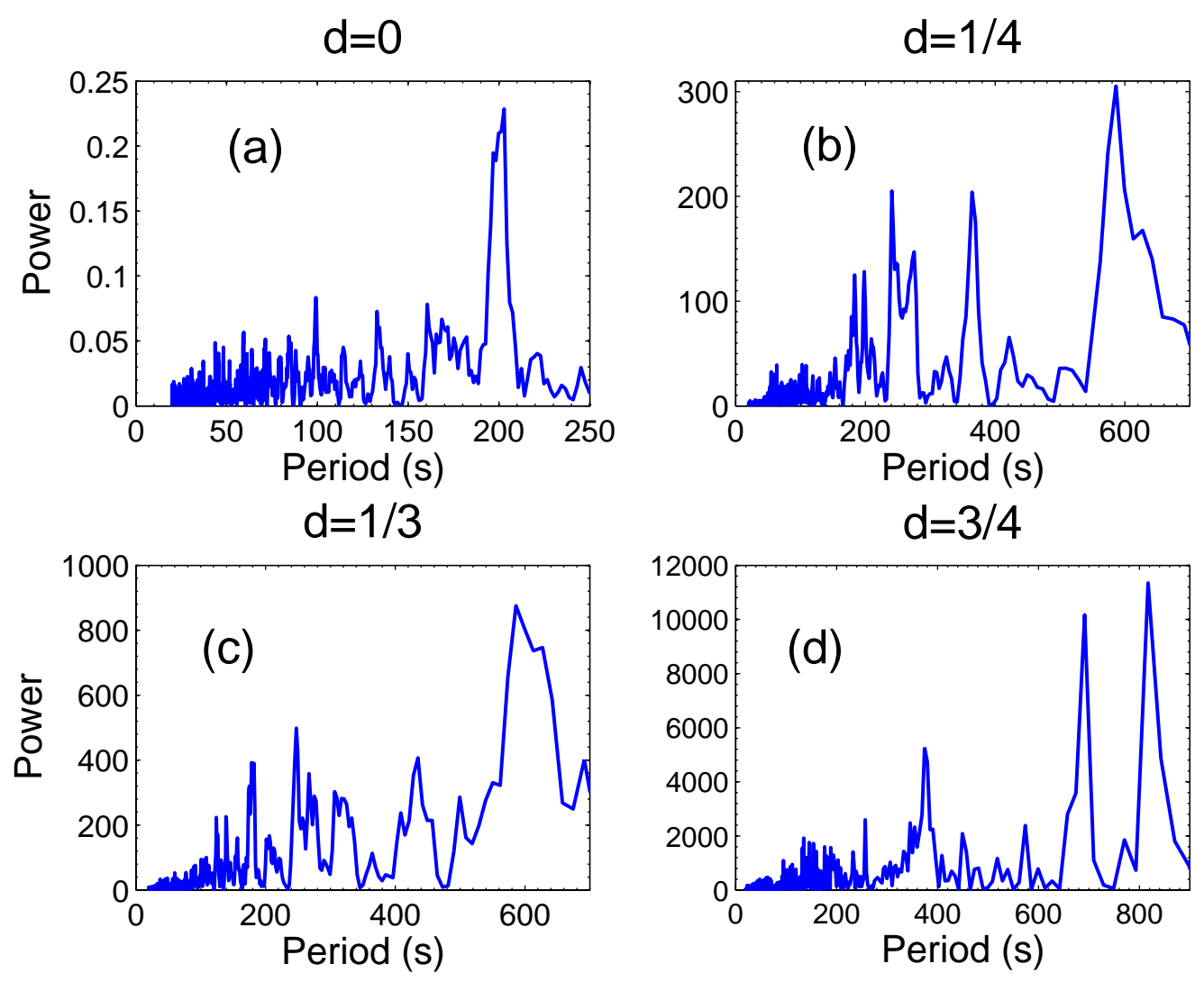

Figure 10: Power spectrum (the squared amplitude of the Fourier transform) vs. period (reverse of the frequency) of radar reflectivity $\eta(t)$ at various depths into cloud relative to cloud top for cirrus $A$ (a) $d=0$, (b) $d=1 / 4$, (c) $d=1 / 3$, (d) $d=3 / 4$. The power peaks are at $\omega_{1}=200 \mathrm{~s}$ in (a), at $\omega_{2}=600 \mathrm{~s}$ in both (b) and (c), and at $w_{3}=820 \mathrm{~s}$ and $w_{4}=700 \mathrm{~s}$ in (d).

Furthermore, it is of interest to know the wind speed in order to calculate the wavelength of the waves that cause these periodic fluctuations in radar reflectivity in the upper $33 \%$ and at depth of $3 / 4$ of cirrus $A$. Because there is a stable thermodynamic stratification in cirrus $A$ as shown in Fig. 1, it is expected that what we find are gravity waves and we could calculate their wavelengths.

ARM DOE archive contains data collected with Balloon-borne sounding system (BBSS). We searched the archive and found that there were three sondes launched on April 19, 2004 at times 5:33, 11:33, and 23:29 UTC, respectively. The launching at 5:33 UTC is relevant to cirrus $A$, while the launching at 11:33 UTC is relevant to cirrus $B$. Unfortunately, the balloon soundings at 5:33 UTC end at height around $9 \mathrm{~km}$ which prevents us to obtain the periodicities in the upper $33 \%$ in terms of wavelength. If we assume a standard wind speed of around $20-30 \mathrm{~m} / \mathrm{s}$ the wavelngths at the top would be $4-6 \mathrm{~km}$, and $12-18 \mathrm{~km}$ at depths $d=1 / 4$, and $1 / 3$. We estimated from the soundings that the wind speed is around $35 \mathrm{~m} / \mathrm{s}$ at depth $d=3 / 4$. The corresponding wavelengths of the gravity waves at depth $d=3 / 4$ are $29 \mathrm{~km}$ and $24 \mathrm{~km}$ for $w_{3}=820 \mathrm{~s}$ and $w_{4}=700 \mathrm{~s}$, respectively. 
These spatial scales indicate that gravity waves occur in cirrus $A$ on larger scales simultaneously with the processes taking place in the generating cells. The generating cells typically exist in the upper $33 \%$ of the cloud but at scales of $1 \mathrm{~km}$. The values of the periods $w_{1}$ and $w_{2}$ are much larger than the scale $3 \tau_{\min }=60 \mathrm{~s}$ of the generating cells at which the drift and diffusion coefficients are obtained from the moments of conditional probability distribution functions, Eq. (4). Therefore, we believe that the periodicities do not influence in any way the derivation of $\tilde{D}_{1}^{\text {eff.d. }}(x)$ and $\tilde{D}_{2}(x)$.

\section{Summary}

In this paper we outline a stochastic approach to investigate the internal structure of radiative properties of cirrus clouds based on empirical modeling and draw conclusions about cirrus dynamical properties in the context of the state of the large-scale atmosphere.

Based on differences in both the state of the large-scale atmosphere, e.g. synoptic low pressure at the surface from 0 to 7.5 UTC and high pressure from 7.5 to 21 UTC, and in thermodynamic stratification we parcel out the cirrus cloud observed on April 19, 2004 at DOE ARM Southern Great Plains facility in two parts, e.g. cirrus $A$ and cirrus $B$, respectively (see Table 2 on page 47 ).

In order to sample thoroughly the internal structure of cirrus properties we consider time series of MMCR radar reflectivity $\eta(t)$ at various depths $d$ into cloud relative to cloud top for both cirrus $A$ and $B$. We find that the probability distribution functions $p(\Delta x, \Delta t)$ of $\eta(t)$ are time-dependent and non-Gaussian for wide range of delay times $\Delta t$ at each depth level for both cirrus $A$ and $B$.

The pdfs of the radar reflectivity $\eta(t)$ in cirrus $A$ for delay times $\Delta t=2 \mathrm{hr}$ exhibit behavior that is consistent with the structure of cirrus based on aircraft in situ measurements [Heymsfield and Miloshevich, 1995] and with results from ground-based Raman lidar studies of cirrus [Comstock et al., 2004]. Note that for a typical wind speed of around $15 \mathrm{~m} / \mathrm{s}$, the $2 \mathrm{hr}$ scale corresponds to approximately $100 \mathrm{~km}$. For delay times $\Delta t=2 \mathrm{hr}$ the upper $33 \%$ of cirrus $A$ shows behavior that is in accordance with what is expected from a region of nucleation with long deposition times for vapor over condensed ice as found in model studies [Khvorostyanov and Sassen, 1998]. The tails of the pdfs of $\eta$ for time lag $\Delta t=30 \mathrm{~min}, 1,2 \mathrm{hr}$ at depths $d=2 / 3$, and $3 / 4$ are close to Gaussian distribution which can be interpreted to be related to the system becoming more stable. The results for the pdf of $\eta(t)$ for delay times $\Delta t=2 \mathrm{hr}$ at the cloud base $d=1$ suggest that most probably sedimentation and/or sublimation took place as expected for this region of cirrus [Heymsfield and Miloshevich, 1995, Comstock et al., 2004] (see Table 2).

In contrast, the tails of the pdfs in cirrus $B$ do not show the structure of properties that is distinctive for cirrus $A$. This difference in the structure of dynamical properties between cirrus $A$ and $B$ can be attributed to different synoptic scale dynamics.

Furthermore, we apply a stochastic approach to study the structure of cirrus properties at much smaller scales, at the scale of $(60 \mathrm{~s}) 1-2 \mathrm{~km}$ which is the scale of the generating cells. We find that the evolution of the time-dependent probability distribution functions is governed by the Fokker-Planck equation with linear drift $\left(D_{1}^{\text {eff.d. }}(x)\right)$ and stochastic 
diffusion with state-dependent, multiplicative noise (quadratic $D_{2}(x)$ ).

From the functional dependence of the drift and the diffusion coefficients using moments of conditional probability distribution functions of the observations we find the specific values of the linear effective drift coefficient $D_{1}^{\text {eff.d. }}(x)$ and the diffusion coefficient $D_{2}(x)$. Therefore, we derive a stochastic model for the radar reflectivity at each depth of the cloud relative to cloud top. The drift coefficient can be tentatively identified with the large-scale deterministic forcing. We find that larger values of the effective drift $\tilde{D}_{1}^{\text {eff.d. }}$ at the top and base of cirrus $A$ versus the middle $50 \%$ characterize the deterministic forcing, while such strong influence of the large-scale dynamics is found only at the base of cirrus $B$ (Figs. 6a,b).

The diffusion coefficient characterizes the small scale, fast stochastic processes in the system and can be associated with in-cloud circulation. Obtained quadratic dependence of $D_{2}(x)$ leads to the existence of a noise-induced drift term $D_{1}^{n . i .}(x)$. The noise-induced drift presents the stochasticity in the dynamics of the system, the influence of the small-scale noise on the slow, large-scale deterministic processes.

We find that both the upper and lower $25 \%$ of cirrus $A$ and both upper $33 \%$ and lower $<25 \%$ of cirrus $B$ are characterized by values of the noise-induced drift, that are relatively small in comparison to the middle $50 \%$ of the cloud. The larger values of the noise-induced drift obtained for the middle portion of both cirrus $A(d=1 / 3,1 / 2$, and $2 / 3)$ and $B(d=1 / 2,2 / 3$, and $3 / 4)$ are in accordance with what is expected from the ice crystal growth and deposition region. A comparison of the values of the noice-induced drift $\tilde{D}_{1}^{n . i .}$ in the middle $50 \%$ of cirrus $A$ versus the middle $50 \%$ of cirrus $B$, reveals larger values for cirrus $A$ which is in accordance with what is expected from intensive ice producing generating cells in $A$ versus somewhat decaying cirrus $B$ (Fig. 7a,b and Table $2)$.

We demonstrate that it is very likely that turbulence takes place in the layer at depth of cloud $d=3 / 4$ in cirrus $B$ that is characterized by neutral stratification (Table 1,2 ).

Finally, we find periodicities in the autocorrelation functions of $\eta(t)$ in cirrus $A$ and no periodicities in cirrus $B$ at intermediate scales. The period (reverse frequency) of fluctuations of radar reflectivity is $w_{1}=200 \mathrm{~s}$ at the top of the cloud and $w_{2}=600 \mathrm{~s}$ at depths $d=1 / 4$, and $1 / 3$, and $w_{3}=820 \mathrm{~s}$ and $w_{4}=700 \mathrm{~s}$ at depth $d=3 / 4$. For wind speed of 10-20 m/s these temporal scales would correspond to spatial scales $2-4 \mathrm{~km}$ at the top and 6-12 km for depths $d=1 / 4$, and $1 / 3$, and $8-16 \mathrm{~km}$ for depth $d=3 / 4$. These spatial scales are suggestive of internal gravity waves that occur on the upper $33 \%$ and at depth of $3 / 4$ of cirrus $A$. The hypothesis of existence of gravity waves is supported by the stable thermodynamic stratification found in cirrus $A$. The absence of periodicities and the neutral stratification are associated with convection from about 9 to $11 \mathrm{~km}$ during cirrus $B$ (see Fig. 1 and Table 2).

Even though based on a single cirrus cloud our study outlines at least initial answers to the problem of the coupling between the structure of cirrus cloud properties and the state of the large-scale atmosphere. Furthermore, it demonstrates the potential of this research to trace back the structure of cirrus properties to cirrus formation mechanisms. The results from such a research would provide recommendations to the climate models to treat cirrus differently owing to different synoptic and/or other different physical forcing. 


\section{References}

[Ackerman and Stokes, 2003] Ackerman, T.P., and G.M. Stokes (2003), The Atmospheric Radiation Measurment Program, Physics Today, 56, 38-43.

[Berner, 2005] Berner, J. (2005), Linking nonlinearity and non-Gaussianity of planetary wave behavior by the FokkerPlanck equation, J. Atmos. Sci., 62, 2098-2117.

[Berner and Branstator, 2005] Berner, J., and G. Branstator (2005), Linear and nonlinear signatures in the planetary wave dynamics of an AGCM: Phase space tendencies, J. Atmos. Sci., 62, 1792-1811.

[Comstock et al., 2004] Comstock, J.M., T.P. Ackerman,and D.D. Turner (2004), Evidence of high ice supersaturation in cirrus clouds using ARM Raman lidar measurements, Geophys. Res. Lett., 31, L11106 doi:10.1029/2004GL019705.

[Cox et al., 1987] Cox, S.K. D.S. McDougal, D.A. Randall, R.A. Schiffer (1987), FIRE - The First ISCCP Regional Experiment, Bull. Amer. Meteor. Soc., 67, 114-118.

[Friedrich and Peinke, 1997] Friedrich, R., and J. Peinke (1997), Description of a turbulent cascade by a Fokker-Planck equation Phys. Rev. Lett., 78, 863-866.

[Friedrich et al., 2000] Friedrich, R., S. Siegert, J. Peinke, St. Luck, M. Siefert, M. Lindemann, J. Raethjen, G. Deuschl, G. Pfister (2000) Physics Letters A, 271, 217-222.

[Friedrich et al., 2002] Friedrich, R., Ch. Renner, M. Siefert, J. Peinke (2002), Comment on Indispensable Finite Time Corrections for Fokker-Planck Equations from Time Series Data, Phys. Rev. Lett., 89, 149401-1

[Frisch, 1995] Frisch, U. (1995), Turbulence, (Cambridge University Press, Cambridge).

[Gardiner, 2004] Gardiner, C.W. (2004), Handbook of Stochastic Methods, Springer-Verlag, Berlin, 3rd edition.

[Gultepe and Starr, 1995] Gultepe, I. and D. O'C. Starr (1995), Dynamical structure and turbulence in cirrus clouds: Aircraft observations during FIRE, J. Atmos. Sci., 52, 4160-4181.

[Gultepe et al., 1995] Gultepe, I., D.O'C. Starr, A.J. Heymsfield, T. Uttal, T.P. Ackerman, and D.L. Westphal (1995), Dynamical characteristics of cirrus clouds from aircraft and radar observations in micro and meso-gamma scales, J. Atmos. Sci., 52, 4060-4078.

[Heymsfield, 1975] Heymsfield, A. J. (1975), Cirrus uncinus generating cells and the evolution of cirriform clouds. Part I: Aircraft observations of the growth of the ice phase, J. Atmos. Sci., 32, 799-808.

[Heymsfield and Miloshevich, 1995] Heymsfield, A.J., and L. M. Miloshevich (1995), Relative humidity and temperature influences on cirrus formation and evolution: Observations from wave clouds and FIRE II, J. Atmos. Sci., 52, 4302-4326.

[Ivanova et al., 2003] Ivanova, K., T.P. Ackerman, E.E. Clothiaux, P.Ch. Ivanov, H.E. Stanley, M. Ausloos (2003), Time correlations and $1 / \mathrm{f}$ behavior in backscattering radar reflectivity measurements from cirrus cloud ice fluctuations, J. Geophys. Res., 108 (D9), 4268-4282. 
[Ivanova et al., 2006] Ivanova, K., H.N. Shirer, and E.E. Clothiaux (2006), Internal variability and pattern identification in cirrus cloud structure: The Fokker-Planck equation approach, J. Geophys. Res., 111, D07203, doi:10.1029/2005JD006364.

[Ivanova and Ackerman, 2007] Ivanova, K. and T.P. Ackerman (2007), Stochastic analysis of neutrally stratified cirrus layer, J. Atmos. Solar-Terrestrial Phys., 69, 2265-2278.

[Khvorostyanov and Sassen, 1998] Khvorostyanov, V. I., and K. Sassen (1998), Cirrus cloud simulation using explicit microphysics and radiation. Part II: Microphysics, vapor and ice mass budgets, and optical and radiative properties, J. Atmos. Sci., 55, 1822-1845.

[Kollias et al., 2007] Kollias, P., E.E. Clothiaux, M.A. Miller, B.A. Albrecht, G.L. Stephens, and T.P. Ackerman (2007), Millimeter-wavelength radars. New frontier in atmospheric cloud and precipitation research, Bull. Amer. Meteor. Soc., 88, 1608-1624.

[Mace et al., 2006] Mace, G. G., et al. (2006), Cloud radiative forcing at the Atmospheric Radiation Measurement Program Climate Research Facility: 1. Technique, validation, and comparison to satellite-derived diagnostic quantities, J. Geophys. Res., 111, D11S90, doi:10.1029/2005JD005921.

[Moran et al., 1998] Moran, K.P., B.E. Martner, M.J. Post, R.A. Kropfli, D.C. Welsh, and K.B. Widener (1998), An unattended cloud-profiling radar for use in climate research, Bull. Amer. Meteor. Soc., 97, 443-455.

[Penland, 1996] Penland, C. (1996), A stochastic model of IndoPacific sea surface temperature anomalies, Physica D, 98, 534-558.

[Penland, 2003] Penland, C. (2003), Noise out of chaos and why it won't go away, Bull. Amer. Meteor. Soc., 84, 921-925; Penland, C. (2003), A stochastic approach to nonlinear dynamics, Bull. Amer. Meteor. Soc., 84, ES43-ES52.

[Quante and Brown, 1992] Quante, M. and P.R.A. Brown (1992), Turbulence in different types of cirrus clouds, In: Proceedings of the 11th ICCP, 10-14 August, Montreal, Atmospheric Environment Service, Toronto, Canada, vol. I, pp. 510-513.

[Renner et al., 2001] Renner, Ch., J. Peinke, and R. Friedrich (2001), Experimental indications for Markov properties of small-scale turbulence, J. Fluid Mechanics, 433, 383-409.

[Risken, 1984] Risken, H. (1984), The Fokker-Planck Equation. Springer-Verlag, Berlin.

[Sardeshmukh and Sura, 2007] Sardeshmukh, P. D., and P. Sura, (2007), Multi-scale impacts of variable heating in climate, J. Climate,20, 5677-5695.

[Sassen et al., 1990] Sassen, K., C.J. Crund, J.D. Spinhirne, M. Hardesty, and J.M. Alvarez (1990), The 27-28 October 1986 FIRE cirrus case study: A five lidar overview of cloud structure and evolution, Mon. Wea. Rev., 118, 2288-2311.

[Sassen et al., 1995] Sassen, K., D.O'C. Starr, G.G. Mace, M.R. Poellot, S.H. Melfi, W.L. Eberhard, J.D. Spinhirne, E.W. Eloranta, D.E. Hagen, and J. Hallet (1995), The 5-6 December 1991 FIRE IFO II jet stream cirrus case study: Possible influence of volcanic aerosols, $J$. Atmos. Sci., 51, 97-123 
[Starr, 1987] Starr, D. O'C. (1987), A cirrus-cloud experiment: Intensive field observations planned for FIRE, Bull. Amer. Meteor. Soc., 68, 119-124

[Starr and Cox, 1985a] Starr, D. O'C. and S. K. Cox (1985a), Cirrus clouds, Part I: A cirrus cloud model, J. Atmos. Sci., 42, 2663-2681.

[Starr and Cox, 1985b] Starr, D. O'C. and S. K. Cox (1985b), Cirrus clouds, Part II: Numerical experiment on the formation and maintenance of cirrus, J. Atmos. Sci., 42, 2682-2694.

[Starr and Quante, 2002] Starr, D. O'C., and M. Quante (2002), Dynamical processes in cirrus clouds: Concepts and models, In: Cirrus, Eds. D.K. Lynch, K. Sassen, D. O'C. Starr, G. Stephens, Oxford University Press, New York.

[Stephens, 1983] Stephens, G.L. (1983), The influence of radiative transfer on the mass and heat budgets of ice crystals falling in the atmosphere, J. Atmos. Sci., 40, 1729-1739.

[Sura and Barsugli, 2002] Sura, P., and J. Barsugli (2002), A note on estimating drift and diffusion parameters from timeseries, Physics Lett. A, 305, 304-311.

[Sura et al., 2005] Sura, P., M. Newman, C. Penland, and P. Sardeshmukh (2005), Multiplicative noise and non-Gaussianity: A paradigm for atmospheric regimes?. J. Atmos. Sci., 62, 1391-1409.

[Tetuya et al., 2007] Tetuya, K., T. Naoko, M. Kazuko (2007), Simple simulation model of the generation of clouds by the flow over an isolated mountain, Nat. Sci. Report, 57, 57-69.

[Weisheimer et al., 2001] Weisheimer, A., D. Handorf, and K. Dethloff (2001), On the structure and variability of atmospheric regimes in coupled climate models, Atmos. Sci. Lett., 2, 72-80. 


\section{Stochastic analysis of neutrally stratified cirrus layer}

Neutrally stratified layers of cirrus clouds are known to be associated with the existence of generating cells. Therefore, it is of interest to describe and quantify the structure of the cloud-generating layer in cirrus through empirical analysis of radar reflectivity observations based on the FPE method. In Ivanova and Ackerman (2007a) we apply the Fokker-Planck equation approach to MMCR measurements from the neutrally stratified layers in cirrus.

Two cirrus cloud systems observed during the winter of 2001 at the ARM Southern Great Plains site are studied because of the distinct neutrally stratified layers formed within the clouds. These two cirrus cloud cases, January 16 and on February 13, and 14 are chosen from a dataset of about twenty cirrus cloud cases that have duration of 6 hr or more and which were observed during the six-month period from January 1 to June 30, 2001 at the ARM SGP site. Most of the cases in the dataset are predominately stably stratified thus not exhibiting noticeable neutral layer.

In order to identify the neutrally stratified layers within the cirrus we first assess the thermodynamical stratification. From radiosonde measurements the merged sounding data product is obtained that contains data of the mixing ratio, temperature, and pressure at 65 levels in height up to $16 \mathrm{~km}$ with $1 \mathrm{~min}$ time resolution. These are data available from the ARM archive. We calculate both potential and equivalent potential temperature and identify the neutrally stratified layers. Within the neutrally stratified layers we analyze observations of radar reflectivity obtained with $35 \mathrm{GHz}$ millimeter wavelength radar (MMCR).

We find that the statistics of radiative properties within the neutrally stratified layers is non-Gaussian and time-dependent. The Fokker-Planck equation approach provides suitable framework to treat non-Gaussian, time-dependent probability distribution functions such as those found for the radar reflectivity time series.

We show that the deviations from Gaussianity of radiative properties of the neutrally stratified generating cells layer in cirrus can be modeled by linear stochastically perturbed dynamics with multiplicative noise statistics. Specific values of the leading linear coeffi-

cients describing the effective, deterministic and noise-induced drift of the backscattering cross-section $\eta$ for the neutral layer of the clouds on January 16, 2001 and February 13, and 14, 2001 are shown in Fig. 11 (on page 43). Because the multiplicative noise is often identified with state-dependent variations of stochastic feedbacks from unresolved system components, the derived stochastic model has the potential to be useful for parameterization of cirrus in global circulation models.

\section{An attempt to distinguish dynamical and microphysical contributions to the radiative properties of cirrus clouds at the ARM SGP site}

The problem of unraveling the interwoven dynamical, microphysical and radiative properties in cirrus has been of interest to the research community for many years. In 
Ivanova and Ackerman (2007b) we address this issue through analysis of radar reflectivities observations from cirrus clouds collected at the ARM SGP site (the poster is available from the ARM web site).

In order to unravel to some extent the interwoven dynamical, microphysical and radiative properties of the cloud we consider time series of radar reflectivities having their magnitude limited within certain $\mathrm{dB}$ range. By doing that, we eliminate one of the unknowns whish is the radiative properties. This allows us to assess the contributions of dynamics and microphysics to the radiative properties in the context of cirrus structure. Furthermore, we aim at distinguishing between these two contributions. Radar reflectivities measured with the DOE MMCR radar have temporal resolution $10 \mathrm{~s}$. Such high temporal resolution makes it possible to conduct the statistical analysis at the scales of the generating cells, which is of order of $1-2 \mathrm{~km}$. We couple these extremely high quality, high resolution and continuous data with method of analysis that has the ability to extract the equation that govern the time evolution of the probability distribution functions through empirical analysis. This is the Fokker-Planck equation method. We apply the FPE method to previously selected time series of radar reflectivities having their magnitude limited within certain $\mathrm{dB}$ range for five different $\mathrm{dB}$ ranges, which covers the cirrus clouds and quantifies the internal structure of cirrus. The outputs of the analysis are the values of the drift and diffusion coefficients of the FPE for each layer characterized with a certain $\mathrm{dB}$ range.

We find that the dynamical contributions to the radiative properties, as expressed by the drift coefficient increase from top of the cirrus downward with increasing both the layer mean radar reflectivity and the layer mean effective radius. We find that the microphysical contributions to the radiative properties are approximate constant from top of the cirrus downward, which is for increasing both the layer mean radar reflectivity and the layer mean effective radius.

\section{Atmospheric turbulence as a Markov process}

Results from this research (Ivanova and Ackerman, 2007c) with emphasis on the relationship between the results from the empirical analysis of cirrus radiative properties observed at the ARM SGP site and physical processes that are responsible for producing such properties, were presented at the 23rd International Conference on Statistical Physics of the International Union for Pure and Applied Physics (IUPAP). The paper was part of the session Dynamical systems and turbulence. Presenting this research at conference on statistical physics was on target because of the following.

Statistical physics (sometimes called statistical mechanics) provides a framework for relating the microscopic properties of individual atoms and molecules to the macroscopic or bulk properties, therefore explaining thermodynamics as a natural result of statistics and mechanics at the microscopic level. The Fokker-Planck equation approach is a tool of statistical physics. By applying it to study the internal structure of cirrus, we take advantage of the ability of statistical mechanics to make macroscopic predictions based on microscopic properties as expressed in the time series of radiative properties. 
A principal mechanism of cirrus maintenance is through individual generating cells which are always producing new ice crystals. The neutrally stratified layer in cirrus is usually the cloud-generating layer and this is the layer associated with embedded cells and turbulence. Therefore, it is of interest to analyze empirical data from cirrus in order to investigate the dynamics of the processes involved in the generating cells. In Ivanova and Ackerman (2007c) we present the results from a stochastic analysis of the backscattering cross-section signal of the hydrometeors in the neutrally stratified layer of cirrus clouds. We show that the neutrally stratified layer of cirrus cloud is characterized by non-Gaussian probability distribution functions (pdfs).

From the master equation written for the probability density functions in the form of the Kramers-Moyal expansion

$$
-\frac{\partial p(x, t)}{\partial t}=\sum_{k=1}^{\infty}\left(-\frac{\partial}{\partial x}\right)^{k} D_{k}(x, t) p(x, t)
$$

by multiplying both sides of Eq. (17) from the left by $x^{n}$ and integrating from the left, e.g. $\int_{-\infty}^{+\infty} d x$ one can obtain an equation for the time evolution of the structure functions $<x^{n}>$ ( $x$ are the increments)

$$
-\frac{\partial}{\partial t}<x^{n}>=\sum_{k=1}^{n} \frac{n !}{(n-k) !} \int_{-\infty}^{+\infty} x^{n-k} D_{k}(x, t) p(x, t) d x
$$

From the empirical analysis we found that the drift coefficient is linear $D_{1}(x)=a_{1} x+a_{0}$ and the diffusion coefficient is second order polynomial $D_{2}(x)=b_{2} x^{2}+b_{1} x+b_{0}$ and from Risken (1984) all coefficients $D_{k}=0$ for $k \geq 3$. Therefore, Eq. (18) is reduced to

$$
\begin{aligned}
& -\frac{\partial}{\partial t}<x^{n}>=n \int_{-\infty}^{+\infty} x^{n-1}\left(a_{1} x+a_{0}\right) p(x, t) d x+n(n-1) \int_{-\infty}^{+\infty} x^{n-2}\left(b_{2} x^{2}+b_{1} x+b_{0}\right) p(x, t) d x \\
& -\frac{\partial}{\partial t}<x^{n}>=n\left(a_{1}<x^{n}>+a_{0}<x^{n-1}>\right)+n(n-1)\left(b_{2}<x^{n}>+b_{1}<x^{n-1}>+b_{0}<x^{n-2}>\right) \\
& -\frac{\partial}{\partial t}<x^{n}>=\left(n a_{1}+n(n-1) b_{2}\right)<x^{n}>+\left(n a_{0}+n(n-1) b_{1}\right)<x^{n-1}>+n(n-1) b_{0}<x^{n-2}>
\end{aligned}
$$

We can then apply the Kolmogorov $-4 / 5$ law which is the requirement that the third order structure functions do not change with time

$$
-\frac{\partial}{\partial t}<x^{n}>=0
$$

for $n=3$. Because the coefficients in front of the leading order in each of $D_{1}(x)$ and $D_{2}(x)$ are at least one order of magnitude larger than the coefficients in front of the next $x$-dependence (as shown in Fig. 12b on page 44), the approximate presentation is 


$$
0 \approx\left(n a_{1}+n(n-1) b_{2}\right)<x^{n}>
$$

which for $n=3$ results to $-a_{1}=2 b_{2}$.

Markov properties are found to hold for physically meaningful interval, e.g. about the size of the cloud generating cells. The evolution of the non-Gaussian, time-dependent pdfs is described by the Fokker-Planck equation with linear stochastically perturbed dynamics with multiplicative noise. Following the $4 / 5$ Kolmogorov law we find that turbulence characterizes the lower part of the neutrally stratified cirrus layer as shown in Fig. 12a on page 44. These findings are in accordance with what is expected from an ice crystal growth and deposition region of cirrus.

\section{Dynamical model and nonextensive statistical mechanics of liquid water path fluctuations in stratus clouds}

In recent years, the formalism of nonextensive statistical mechanics, first introduced by Tsallis [J. Stat. Phys., 52, 479 (1988)] and later further developed by others, has gained considerable interest. The new theoretical approach is suitable to treat physical systems of sufficient complexity that cannot maximize the usual Boltzmann-Gibbs-Shannon (BGS) entropy, the latter leading to the usual statistical mechanics. In such a case, the system maximizes some other, more general entropy measure, such as the Tsallis entropies, which have the BGS as a limit. Various reasons may cause some physical systems not to maximize the BGS entropy, e.g. long-range correlations, multifractality or simply the fact that the system is not in equilibrium owing to some external forcing. Recently it has been shown that nonextensive statistical mechanics is particularly useful in describing two-dimensional Eulerian turbulence and the stochastic properties of fully-developed turbulent flows.

In this study, we apply the Beck dynamical model to liquid water path fluctuations in stratus clouds in the framework of nonextensive statistical mechanics. As introduced to describe fully developed turbulence, the Beck dynamical model aims neither to solve the turbulence problem nor to reproduce fully the spatio-temporal dynamics of the NavierStokes equations, but rather to provide a simple model that captures some of the most important statistical properties of the phenomena in an analytically tractable manner.

In classical studies, the phenomenology of turbulence has been described by self-similar cascades, in which an identical, scale-invariant step is repeated from large scales to small ones, as the small ones produce even smaller ones until the turbulent flow energy gets dissipated on the smallest scale. More realistic description of turbulence is achieved by generalization of this approach to anisotropic scaling and multiplicative cascade models. Cascade processes generically give rise to multifractals. The resulting multifractal behav-

ior of a random variable is scale invariant and can be determined either by the scaling of its probability distribution functions or by the scaling of its structure functions. In hydrodynamics, the velocity structure functions are expected to exhibit multi-affine scaling, e.g. nonlinear scaling of the structure function exponents. 
In contrast, the probability distribution functions that are obtained within the nonextensive statistical mechanics approach are not scale invariant [Tsallis, 1988]. The Beck dynamical model in the framework of Tsallis statistics describes the evolution of the timedependent probability distribution functions of a random variable for different delay times. The purpose of this study is to present empirical evidence that the probability distribution functions of the liquid water path fluctuations in stratus clouds are time-dependent and their evolution can be sufficiently well described in the framework of Tsallis statistics.

We show that the model describes well the shape and the tails of the probability distribution functions (see Fig. 13 on page 44). We find that the transition between the small-time-scale model of a nonextensive process and the large-scale Gaussian extensive homogeneous fluctuation model is found to be at around $24 \mathrm{hr}$. The model thus can provide a useful framework for treating liquid water path in global circulation models.

\section{Internal variability and turbulence in cirrus: Fokker-Planck equation approach}

Building on the knowledge developed in previous years, we applied the Fokker-Planck approach to study the internal variability of cirrus clouds (Ivanova, 2006, Ivanova et al., 2006a,b). Cirrus clouds play an important role in the climate system owing to their interwoven microphysical, dynamical, and radiative properties. The problem of separating the dynamical from microphysical contributions in cirrus clouds is a long standing one in the research community. Our research on describing and quantifying the internal variability of cirrus clouds represents noticeable progress toward finding a solution to this problem.

We consider a cirrus cloud case observed on January 26 and 27, 1997 at the ARM SGP site. We analyzed radar reflectivity data collected with the millimeter wave cloud radar that operates at $34.86 \mathrm{GHz}$ and is deployed at this site. Using the radiosonde measurements of temperature, pressure, and ice/water mixing ratio, we first assessed the vertical stratification within the cloud structure. We found that the lower portion of the cloud is characterized by absolutely stable stratification. The middle layer contains sublayers of conditionally unstable and absolutely unstable stratification. Finally, the upper layer is characterized by neutral stratification.

In order to describe the internal structure of the cloud as precisely as possible and in a uniform fashion, we defined sub-layers within each of the two upper layers, the one having neutral and the one having unstable stratification. Furthermore, we defined for each sublayer a time series of the backscattering cross-section that was analyzed for quantification

of the dynamics of their fluctuations. We defined the nine time series of observations to be those measured at the maximum height within each region.

In this study we obtained and analyzed the time-dependent probability distribution functions of each of the above-defined time series from the bulk of the cirrus cloud. We used the Fokker-Planck equation approach for studying the time-dependent crosscorrelations between the fluctuations of these time series in regions of the cloud that have different stratification. By doing so, we were able to quantify the internal variability in layers having different stratification within the cirrus cloud. 
We found that the drift term of the Fokker-Planck equation gradually increases from the top of the cloud downward within the neutral layer and slightly increases within the unstable layer. The diffusion term has approximately constant values within the neutral layer and slightly increasing values in the unstable layer. These findings suggest that the deterministic part of the dynamics gradually increases from the top of the cloud downward, which is an expected result in view of an increasing "restoring force" due to an increased mass of the particles.

The specific values of the drift and diffusion terms in the neutral layer satisfy the relation that holds true for fully developed turbulence according to the $4 / 5$ Kolmogorov law as shown in Fig. 14 on page 45). This result is in agreement with the likelihood that fully developed turbulence takes place in a well-mixed layer having neutral stratification, as occurs in the neutral layer. This is a very important result showing that we can distinguish regions with different dynamical properties such as turbulence from the more passive parts of cirrus, based on stochastic analysis of radiative properties.

We see two avenues for using these findings to facilitate improved parameterization of subgrid processes in cloud-resolving and general circulation models. One is further research on tying the drift and diffusion coefficients of the Fokker-Planck equation that governs the time-dependent probability distribution functions of the backscattering cross section signals to the evolution of the particle size distribution that produce them. This is a long-term goal in our research. The other avenue is for modelers to use the FokkerPlanck equation approach to compare the values for the drift and diffusion coefficients of the modeled backscattering cross section with those of observations reported in this study, with the aim of improving the formulation of the parameterizations used in general circulation models.

The approach described in this section has much value for a broader investigation of typical, characteristic patterns of internal variability in cirrus clouds. We intend to pursue this research further.

\section{Internal variability dependence in cirrus cloud structure}

In Ivanova et al., (2005a,b) we report some preliminary results on distinguishing various vertical regions in cirrus cloud that are apparently dominated by different physical phenomena. We analyze radar reflectivity observations measured with the DOE MMCR radar at the SGP site. First, we take advantage of the fact that the three quantities measured with the $35 \mathrm{GHz}$ millimeter wave radar the radar reflectivity, the Doppler velocity, and the Doppler spectral width, are not independent of one another. That is, the Doppler velocity is the reflectivity-weighted mean particle fall velocity and the Doppler spectral width is its reflectivity-weighted standard deviation, or Doppler spread or Doppler broadening.

Therefore, if we identify regions in the cloud having approximately constant values of radar reflectivity (i.e. backscattering cross section) or values within a certain range, then, from a mathematical point of view, we could eliminate the weighting factor. As a result, the interpretation from a physical point of view given by any further analysis of 
the mean velocity and its standard deviation will become more accurate, more straightforward, and more clear. Such partitioning of the regions will facilitate our successfully surmounting the problem of interwoven cloud dynamical and microphysical contributions during subsequent data analysis. Such a separation will allow us to specify how the roles of atmospheric dynamics and microphysics vary within cloud structure during future data analysis. Furthermore, we show that this also leads to the identification of regions in the cloud where different types of stratification exist and where different physical processes are dominant.

In order to make an initial estimate of the regions in the cloud as given by radar reflectivity within certain limits, we start by dividing the $[-45,+10] \mathrm{dB}$ range of the cloud radar reflectivity into subintervals. We find that radar reflectivity values within the [$45,-5] \mathrm{dB}$ range occupy predominately the upper two thirds of cirrus while the data in the $[-5,+10] \mathrm{dB}$ range exist during shorter period of time and occupy the lower region of cirrus. Our hypothesis is that different processes are taking place in these two regions of the cloud.

To test our hypothesis, we studied the consequences of the vertical stratification in cirrus clouds through contour maps of equivalent potential temperature calculated from the radiosonde measurements of temperature, pressure, and ice/water mixing ratio available from the ARM archive.

We find that the occurrence of neutral stratification coincides with the upper part of the cloud, where comparable contributions from particles with similar sizes is more likely (see Fig. 15 on page 45). The next layer from the top downward is the unstable region in the cirrus cloud which coincides with the convective region in the cloud where it is more likely that the largest particle size contribution dominates the radar reflectivity. Finally, further downward a stable region is identified via the positive vertical derivative of the equivalent potential temperature that we found to coincide with the region that has been identified by radar reflectivity values within the $[-5,+10] \mathrm{dB}$ range. Our findings are in agreement with previous work on cirrus clouds by Gultepe and Starr (J. Atmos. Sci.,52, 4159, 1995)

\section{Time-dependent distribution of liquid water path in stratus clouds}

Knowledge of the spatio-temporal distribution of liquid water is of paramount importance for the development of improved parameterizations in global circulation models. Substantial progress has been made (Ivanova 2004, Ivanova et al., 2004b) both in investigating at different time delays the time-dependent distributions of fluctuations of the liquid water path in stratus clouds and in deriving an underlying mathematical (statistical or model-free) equation the Fokker-Planck equation that governs them. This is achieved through empirical analysis of the liquid water path (LWP) measured using microwave radiometer on January 9-14, 1998 during which period a continuous stratus cloud is observed at the SGP ARM site. The LWP signal is non-stationary with highly irregular and clustered fluctuations owing to a set of various physical influences that occur over different temporal and spatial scales. Thus, it is of interest to both distinguish and quantify from 
first principles the deterministic and stochastic influences on the LWP signal in stratus clouds.

It is known that two equivalent master equations govern the dynamics of a system, i.e. the Fokker-Planck equation and the Langevin equation, the former for the probability distribution function of temporal and spatial signal increments, the latter for the increments themselves. Therefore, starting from LWP observations, we obtained both the Langevin equation that represents the change with time of the increments of the LWP signal and the Fokker-Planck equation that governs the time-dependent distributions of the LWP from small to large time scales. A drift term and a diffusion term describing the deterministic and stochastic influences on the non-Gaussian tails of the liquid water probability distributions were obtained from empirical data.

Working on the problem of identifying and describing the random and deterministic contributions to the liquid water path signal had a twofold value for continued progress in our research program. One is due to the contribution of this study to incorporating the effects of the spatiotemporal distribution of liquid water into global circulation models. The other is the importance of developing this methodology and making its basic version available for application to the study of the internal variability of other quantities in other types of clouds. We intend to use this approach extensively for future research in studying the internal variability of cirrus clouds using the observations available from the huge ARM database.

\section{Local correlations and multifractal behavior in marine boundary layer cloud dynamics}

The purpose of this research is to make progress on the development and improvement of methodology to tackle the problem of multifractality in time series (Kitova et al., 2004). The approach is based on the multifractal research that we have conducted during the years after our initial study on the multifractal analysis of liquid water path in stratus clouds (Ivanova and Ackerman, Phys. Rev. E, 59, 2778, 1999). We analyze cloud base height $(\mathrm{CBH})$ data measured at the Azores Islands during the Atlantic Stratocumulus Transition Experiment (ASTEX). The dynamics of the CBH evolution is determined by variety of processes in the atmosphere.

We present an analysis of the local correlations and the multi-affine properties of $\mathrm{CBH}$ with respect to their diurnal evolution. In order to test how the scaling properties of the $\mathrm{CBH}$ time series change along its length, we applied the DFA method within an observation box with width size $\mathrm{w}$ of $7 \mathrm{~h}$ that is moving with a step $? \mathrm{w}=30 \mathrm{~min}$ from the beginning toward the end of the data. We find that local correlations hold with well established day-night evolution of local similarity exponent that is related to dominant physical processes. Furthermore, the multi-affine structure of the CBH data is demonstrated by the sets of local roughness exponents. 


\section{Time correlations in cirrus clouds and internal variability at isodepths of the cloud}

Substantial progress has been made in investigating, interpreting, and understanding the spatial pattern of time correlations in cirrus clouds. We first focused our efforts on answering the question of whether or not the parameters characterizing the time correlations of the fluctuations of radar reflectivity and its two moments in cirrus clouds differ depending on the position in the cloud that is, at the top or the bottom or at three levels in the bulk of the cloud (Ivanova et al., 2004a). We apply the detrended fluctuation analysis (DFA) method to characterize the correlations in these signals. The DFA method is suited to accurately quantifying power-law correlations in noisy nonstationary signals with polynomial trends. The advantage of the DFA method over conventional methods, such as the power spectrum analysis, is that it avoids the spurious detection of apparent long-range correlations that are an artifact of the nonstationarity (related to linear and higher order polynomial trends in the data).

Using the detrended fluctuation analysis method, we found that in all cases, powerlaw time correlations exist with a crossover between regimes occurring at about 3 to 5 minutes. To verify the robustness of our results, we tested them against surrogate time series that were obtained through randomly shuffling the amplitudes of the backscattering cross section, Doppler velocity, and Doppler spectral width. We found that the long-range correlations do vanish in the surrogate data, which further supports the validity of our results.

We also found that the short-time-range correlations of radar backscattering cross section are uncorrelated, i.e. they are Brownian-like; at time lags longer than about 3 to 5 minutes, the correlations are of the $1 / \mathrm{f}$ - noise type. Furthermore, certain asymmetry exists in the respective scaling properties at the top and the bottom layers and within the bulk of the clouds. This asymmetry is interpreted to be due to different physical mechanisms on the boundaries and within the bulk of the cloud, depending on the time scales.

\section{Objective criteria for distinguishing seasons in the western Arctic}

This theme is outside of the original objectives of this proposal, but because of its importance for treating accurately the Arctic cloud system we conducted this study.

One characteristic feature of mixed-phase clouds is that, even though they are observed in all seasons at the NSA ARM site, they occur most frequently during the spring and fall transition seasons (Ivanova et al., 2004c). The definition and duration of the seasons in the Arctic, however, differs from the standard delineation of the seasons for the midlatitudes, which is still widely used. Although such seasonal partitioning works well for the mid-latitude environment, it does not provide a representative seasonal classification for the Arctic.

If seasonal classification is not representative of the Arctic system, then any statistical and correlative analyses for this region may be in error. For example, if some of the 
properties of the spring transition are averaged into the summer-season statistics, then processes that occurred during the rapid melt stage in the Arctic would influence the summer statistics. Hence, inferences that one might draw regarding, for example, the atmospheric/oceanic forcing of Arctic cloud cover in the summer, may be erroneous.

Ultimately, the time periods that one uses for the delineations between seasons must correspond with the physical processes in which one is interested. Therefore, the first step in investigating the Arctic cloud system should be searching for objective criteria to define the seasons in the Arctic. We conducted such a study and reported the results at the ARM Science Team Meeting in March 2004 and we also wrote a paper (Ivanova et al., 2004c, 2006c).

The breakdown of the annual cycle into seasons is an important, although not always quantified, part of many climatologies. The objective of this study is to determine the seasonal periods over which the physical mechanisms affecting cloud properties are similar. Using the mean local variability of daily maximum $T_{\max }$ and minimum $T_{\min }$ temperatures leads to a new methodology for quantifying the average time of the spring and autumn transitions as well as of the summer and winter seasons for the western Arctic. The hypothesis that the minimum temperature variability is associated with the ocean and the maximum temperature variability is associated with the cloudiness/radiation is explored and the data analysis that supports it is presented. The method for determining the date of the summer onset based on the decrease of the $T_{\max }$ standard deviation (as shown in Fig. 16 on page 46) is found to be consistent with the objective radiometric approach discussed elsewhere. The onset of the fall transitional coinsides with the jump in the shortwave albedo.

Similar results are obtained for nine stations across western Arctic and different result is obtained for a station in Siberia, Khatanga, that is not along the coast and is outside the $10{ }^{\circ} \mathrm{C}$ July isotherm and thus is outside the Arctic basin. Although the methodology is based on the variability of the temperature, supporting evidence that results from other physical processes (solar irradiance, snow depth) is found to be consistent with the proposed definition of the seasons. This methodology is shown to be robust, applicable over the western Arctic, and gives results that are consistent with older qualitative climatologies.

This delineation of the seasons will facilitate the process of obtaining statistics of the physical quantities of interest via averaging the observations or model results using an appropriate definition and duration of the seasons. 


\section{Deliverables:}

\section{Peer-Reviewed Journal Papers:}

1. K. Ivanova, T.P. Ackerman, (2008a), Tracking nucleation-growth-sublimation in cirrus clouds using ARM millimeter wavelength radar observations, J. Geophys. Res. (submitted)

2. K. Ivanova, and T.P. Ackerman, (2007a), Stochastic analysis of neutrally stratified cirrus layer,J. Atmos. Sollar-Terrestrial Phys., doi:10.1016/j.jastp.2007.06.009

3. K. Ivanova, H.N. Shirer, T.P. Ackerman, E.E. Clothiaux, (2007), Dynamical model and non-extensive statistical mechanics of liquid water path fluctuations in stratus clouds, J. Geophys. Res., 112, doi:10.1029/2006JD007493

4. K. Ivanova, H.N. Shirer and E.E. Clothiaux, (2006a) Internal variability and pattern identification in cirrus cloud structure: The Fokker-Planck equation approach, $J$. Geophys. Res.,111, D07203, doi:10.1029/2005JD006364

\section{Papers Published in Conference Proceedings:}

5. K. Ivanova, H.N. Shirer, E.E. Clothiaux, (2006b) Internal variability and pattern identification in cirrus cloud structure within the Fokker-Planck equation framework, In: Proc. of the Sixteenth Atmospheric Radiation Measurements (ARM) Science Team Meeting, D. Carrothers, (Ed.), Albuquerque, NM, 2006. pp.1-6. (paper plus poster) http://www.arm.gov/publications/proceedings/conf16/author.stm\#I

6. K. Ivanova, H.N. Shirer, E.E. Clothiaux, (2005a) Internal variability dependence on cirrus cloud structure, In: Proc. of the Fifteenth Atmospheric Radiation Measurements (ARM) Science Team Meeting, D. Carrothers, (Ed.), Daytona Beach, Florida, 2005, pp. 1-5. (paper plus poster) http://www.arm.gov/publications/proceedings/conf15/author.stm\#i

7. K. Ivanova, (2004) Time series analysis of microwave signals. Fokker-Planck equation approach, In: Proceedings of Progress in Electromagnetic Research Symposium, PIERS04, B. Poljak (Ed.), Pisa, Italy, 2004, pp. 69-72. (paper plus talk))

8. K. Ivanova, T.P. Ackerman, H.N. Shirer, E.E. Clothiaux, (2004a) Time correlations in backscattering radar reflectivity measurements from cirrus clouds, In: Proc. of the Fourteenth Atmospheric Radiation Measurements (ARM) Science Team Meeting, D. Carrothers, (Ed.), Albuquerque, NM, 2004, pp. 1-7. (paper plus poster)

http://www.arm.gov/publications/proceedings/conf14/author.stm\#i

9. N. Kitova, K. Ivanova, M. Ausloos, T.P. Ackerman, M.A. Mikhalev, (2004) Local correlations and multifractal behavior in marine boundary layer cloud dynamics, In: Proc. of the Fourteenth Atmospheric Radiation Measurements (ARM) Science Team Meeting, D. Carrothers, (Ed.), Albuquerque, NM, 2004, pp. 1-8. (paper plus poster)

http://www.arm.gov/publications/proceedings/conf14/author.stm\#i 
10. K. Ivanova, T.P. Ackerman, M. Ausloos, (2004b) Equations governing space-time variability of liquid water path in stratus clouds, In: Proc. of the Fourteenth Atmospheric Radiation Measurements (ARM) Science Team Meeting, D. Carrothers, (Ed.), Albuquerque, NM, 2004, pp. 1-8. (paper plus poster)

http://www.arm.gov/publications/proceedings/conf14/author.stm\#i

\section{Contributed papers at International Conferences:}

11. K. Ivanova, and T.P. Ackerman, (2008b) Stochastic approach to study cirrus structure using ARM millimeter wavelength radar observations, Eighteenth Atmospheric Radiation Measurements (ARM) Science Team Meeting, Norfolk, VA, March 10-14, 2008 (poster)

12. K. Ivanova, and T.P. Ackerman, (2007b) An attempt to distinguish dynamical and microphysical contributions to the radiative properties of cirrus clouds at the ARM SGP site, Seventeenth Atmospheric Radiation Measurements (ARM) Science Team Meeting, Monterey, CA, March 26-30, 2007 (poster)

13. K. Ivanova, and T.P. Ackerman, (2007c) Atmospheric turbulence as a Markov process. The 23rd International Conference on Statistical Physics of the International Union for Pure and Applied Physics (IUPAP), Genoa, Italy, July 9-13, 2007 (poster)

14. K. Ivanova, (2006) Turbulence in the upper troposphere. Fokker-Planck equation approach. Third IAGA/ICMA Workshop on Vertical Coupling in the Atmosphere / Ionosphere System, Varna, Bulgaria, September 18-22, 2006. (talk)

15. K. Ivanova, H.N. Shirer and E.E. Clothiaux, (2005b) Internal variability and pattern identification in cirrus cloud structure, European Geophysical Union, Vienna, Austria, April 22-29, 2005. (poster)

16. K. Ivanova, J.Y. Harrington, H. Verlinde, E.E. Clothiaux, C.P. Bahrmann, (2004c) Objective criterion to distinguish seasons in Arctic climate, Atmospheric Radiation Measurements (ARM) Science Team Meeting, Albuquerque, NM, March 22-26, 2004. (poster)

\section{Unpublished manuscripts:}

17. K. Ivanova, J. Verlinde, J.Y. Harrington, E.E. Clothiaux, H.N. Shirer, N.C. Johnson, C.P. Bahrmann, (2006c) Objective criterion to distinguish seasons in the western Arctic

\section{Conferences attended:}

18. Atmospheric Radiation Measurements (ARM) Science Team Meeting, Monterey, CA, March 26-30, 2007

19. Atmospheric Radiation Measurements (ARM) Science Team Meeting, Albuquerque, NM, March 27-31, 2006 
20. Third IAGA/ICMA Workshop on Vertical Coupling in the Atmosphere/Ionosphere System, Varna, Bulgaria, September 18-22, 2006.

21. European Geophysical Union, Vienna, Austria, April 22-29, 2005

22. Atmospheric Radiation Measurements (ARM) Science Team Meeting, Daytona Beach, Florida, March 14-18, 2005

23. Atmospheric Radiation Measurements (ARM) Science Team Meeting, Albuquerque, NM, March 22-26, 2004 

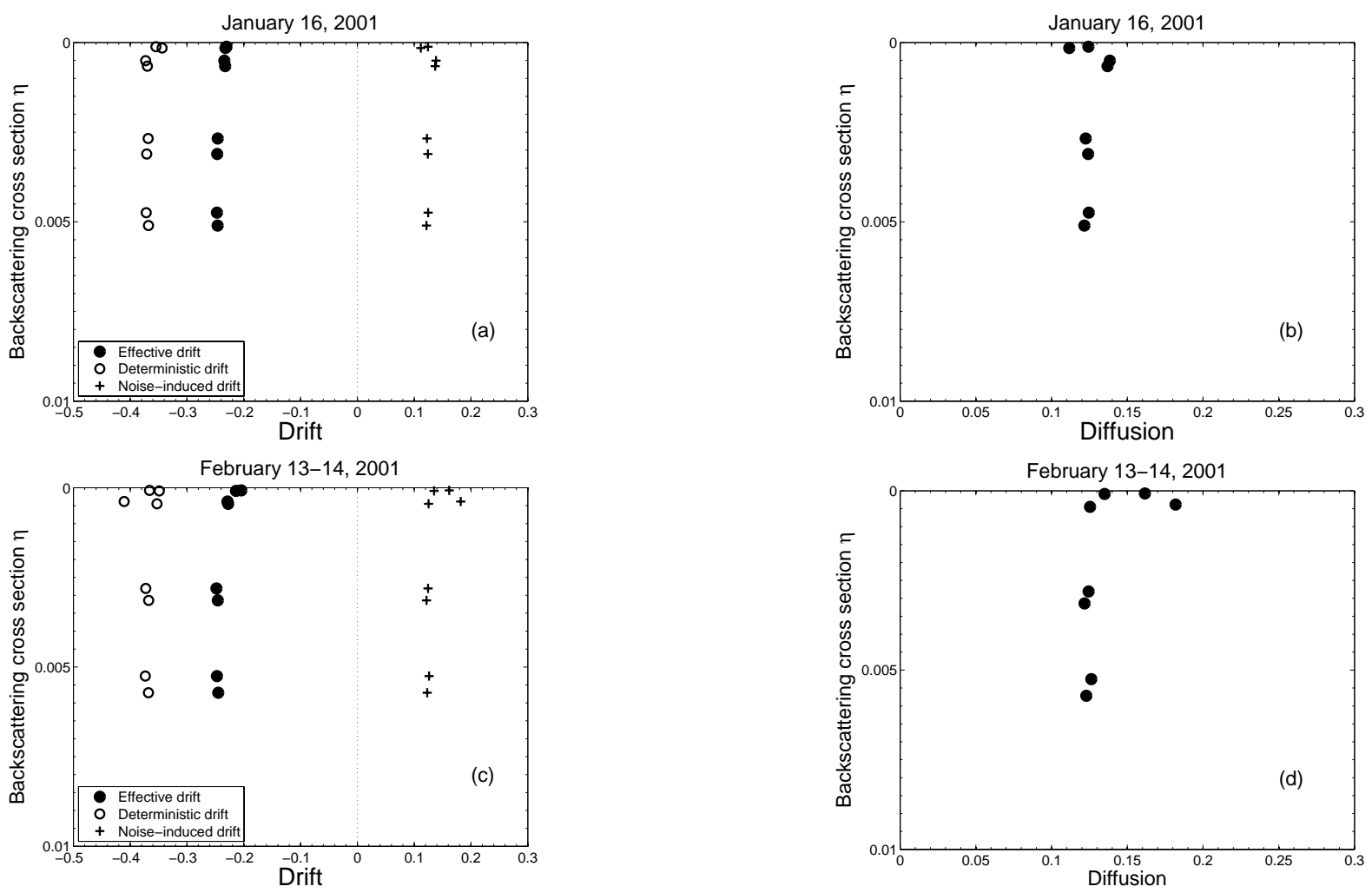

Figure 11: Values of the leading linear coefficients describing the effective, deterministic and noise-induced drift of the backscattering cross-section $\eta$ for the neutral layer of the cloud on (a) January 16, 2001 and (c) February 13, and 14, 2001. Bold dots present the leading linear coefficient $a_{1}$ of the inferred effective drift $\tilde{D}_{1}^{e f f}(x)=a_{1} x+a_{0}$. Plus signs denote the noise-induced drift $\left.\tilde{D}_{1}^{n . i \cdot}(x)=b_{2} x\right)$ owing to the state-dependent multiplicative noise, e.g. nonconstant diffusion. Circles mark values of the linear coefficient $h_{1}$ of derived deterministic dynamics (deterministic drift) $\tilde{D}_{1}^{\text {d.d. }}(x)=h_{1} x+h_{0}$. Values of the leading quadratic coefficient $b_{2}$ describing the diffusion coefficient $\tilde{D}_{2}(x)=b_{2} x^{2}+b_{1} x+b_{0}$ of the backscattering cross-section $\eta$ for the neutral layer of the cloud on (b) January 16, 2001 and (d) February 13, and 14, 2001. 

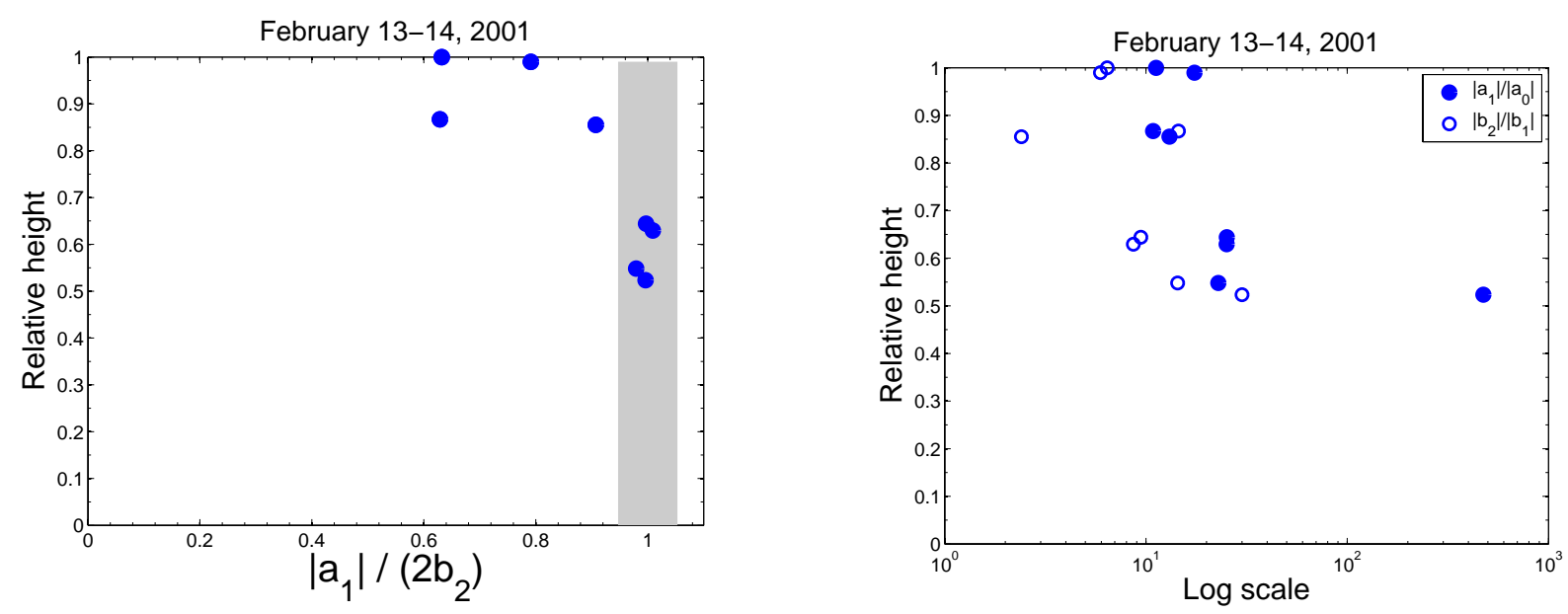

Figure 12: (a)Values of the relation $\left|a_{1}\right| /\left(2 b_{2}\right)$ between the leading linear coefficient describing the effective and noise-induced drift of the backscattering cross-section $\eta$ for the neutral layer of the cloud on February 13, and 14, 2001. In accordance with the 4/5 Kolmogorov law $\left|a_{1}\right| /\left(2 b_{2}\right)=1$ indicates region of turbulence.(b) Values of $\left|a_{1}\right| /\left|a_{0}\right|$ and $\left|b_{2}\right| /\left|b_{1}\right|$ demonstrating that $\left|a_{1}\right| /\left|a_{0}\right|>>1\left|b_{2}\right| /\left|b_{1}\right|>>1$.

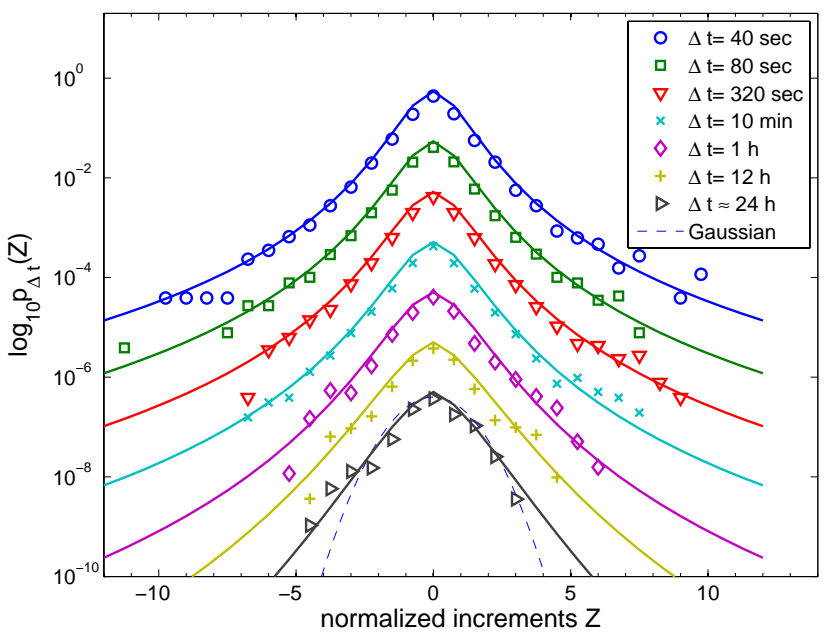

Figure 13: Probability distribution function of normalized increments $Z(t, \Delta t)$ of liquid water path signal (symbols) and the Tsallis-type distribution function (solid curves) for $\Delta t=40 \mathrm{sec}, 80 \mathrm{sec}, 320 \mathrm{sec}, 10 \mathrm{~min}, 1 \mathrm{~h}, 12 \mathrm{~h}$, and $\sim 24 \mathrm{~h}(24 \mathrm{~h} 46 \mathrm{~min} 40 \mathrm{sec})$. The last value of $\Delta t$ is chosen such that at least the PDF for the positive value of the increments converges to a Gaussian distribution (dashed curve). The PDFs (symbols and curves) for each $\Delta t$ are displaced by 10 with respect to the previous one; the top curve for $\Delta t=40 \mathrm{sec}$ is not moved. The values of the parameters for the Tsallis-type distribution function for each $\Delta t$ are summarized in Table 1. 


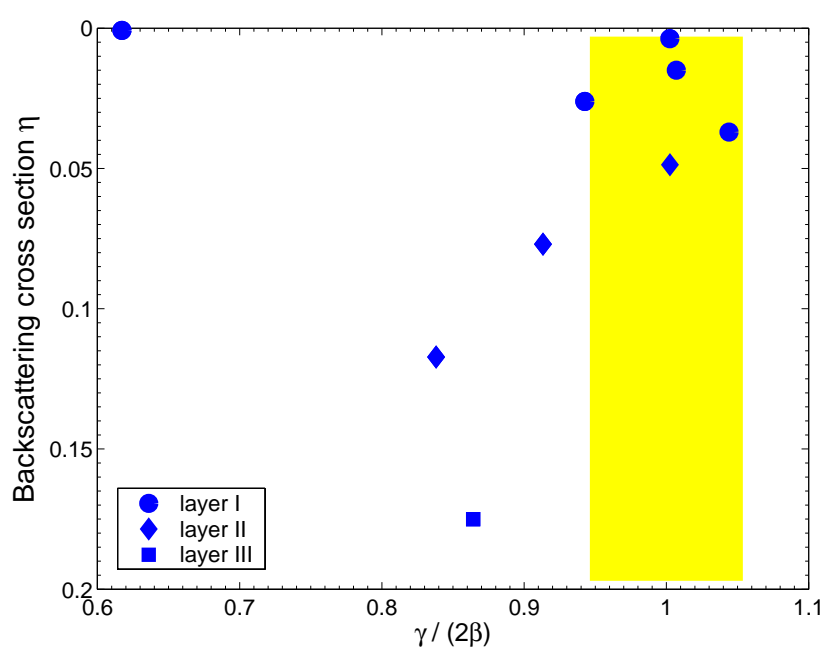

Figure 14: Values of $\gamma /(2 \beta)$ of the backscattering cross-section $\eta$ in the three major layers in the cirrus cloud. Each ordinate value represents the average value of $\eta$ for each of the $\eta$ time series for which the leading drift $\gamma$ and diffusion $\beta$ coefficients are obtained. The reverse order of the ordinate values is intended to give a sense of how the $\gamma /(2 \beta)$ ratio changes with height. For fully developed turbulence, $\gamma /(2 \beta)=1 \pm 0.05$, as indicated by the yellow area.

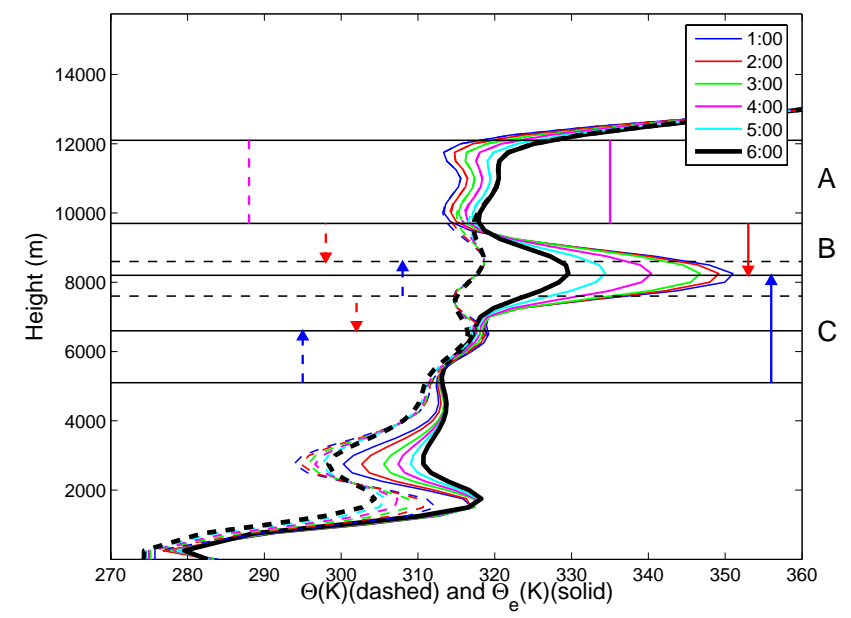

Figure 15: Potential temperature (dashed) and equivalent potential temperature (solid) profiles at 1:00,..,6:00 UTC observed on January 27, 1997 at the ARM SGP site. 

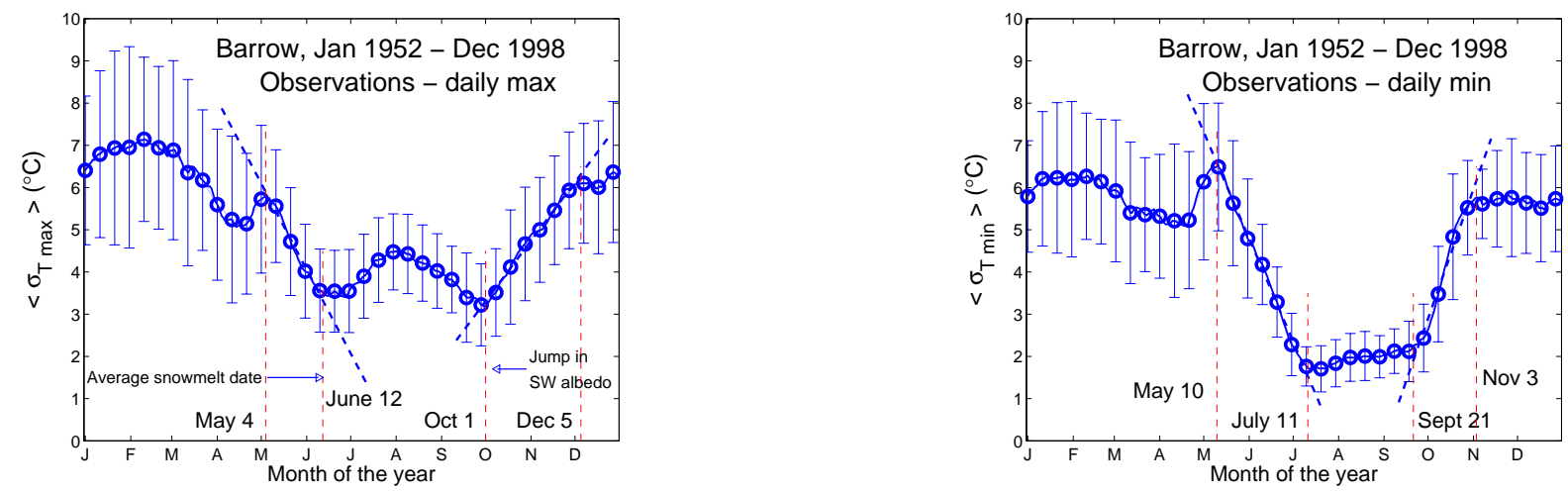

Figure 16: Average running standard deviations of daily maximum $<\sigma_{\mathrm{T}_{\max }}>$ (left panel) and minimum $<\sigma_{\mathrm{T}_{\min }}>$ (right panel) temperatures measured at Barrow from Jan. 1, 1952 to Dec. 31, 1998 (Table 1). The running standard deviation is calculated over a window with duration $w=30$ days. The error bars mark the one $\sigma$ (standard deviation) of $\left\langle\sigma_{\mathrm{T}_{\max }}>\right.$ and $<\sigma_{\mathrm{T}_{\min }}>$ due to taking the average over 44 years. The vertical dashed lines indicate the end of one season and the beginning of the next season. 
Table 2: Summary of the results on identifying the structure of dynamical properties of cirrus based on stochastic analysis of radar reflectivity observations. Results are placed in the context of the synoptic environment and the thermodynamic stratification.

\begin{tabular}{|c|c|c|}
\hline & Cirrus $A$ & Cirrus $B$ \\
\hline $\begin{array}{l}\text { Synoptic } \\
\text { environment }\end{array}$ & $\begin{array}{l}\text { Low pressure system } \\
(\mathrm{L})\end{array}$ & $\begin{array}{l}\text { High pressure system } \\
(\mathrm{H})\end{array}$ \\
\hline \multirow{2}{*}{$\begin{array}{l}\text { Thermodynamic } \\
\text { stratification } \\
\text { (Fig. 1) }\end{array}$} & Stable & $\begin{array}{l}\text { Stable between } d=0(12 \mathrm{~km}) \text { and } \\
d=1 / 4(11 \mathrm{~km})\end{array}$ \\
\hline & & $\begin{array}{l}\text { Neutral between } d=1 / 4(11 \mathrm{~km}) \\
\text { and } d=3 / 4(9 \mathrm{~km})\end{array}$ \\
\hline \multicolumn{3}{|r|}{ ( } \\
\hline \multirow[t]{2}{*}{$\begin{array}{l}\text { Large } \\
2 \mathrm{hr}(\sim 100 \mathrm{~km})\end{array}$} & $\begin{array}{l}\text { Structure of nucleation-growth- } \\
\text { sublimation regions identified } \\
\text { from the pdfs (Fig. } 3 \text { ) }\end{array}$ & $\begin{array}{l}\text { Structure of cirrus properties can } \\
\text { not be identified from the pdfs } \\
\text { (Fig. } 4 \text { ) }\end{array}$ \\
\hline & $\begin{array}{l}\text { - upper } 33 \% \text { - region of nucle- } \\
\text { ation with long deposition times } \\
\text { for vapor over condensed ice } \\
\text { - cloud base - region of sedi- } \\
\text { mentation and/or sublimation }\end{array}$ & \\
\hline $\begin{array}{l}\text { Intermediate } \\
4-29 \mathrm{~km}\end{array}$ & $\begin{array}{l}\text { Gravity waves (Figs. 9a,b, 10) } \\
- \text { top }-w_{1}=200 \mathrm{~s}(4-6 \mathrm{~km}) \\
- \text { upper } 33 \%-w_{2}=600 \mathrm{~s}(12- \\
18 \mathrm{~km}) \\
-d=3 / 4-w_{3}=820 \mathrm{~s}(29 \mathrm{~km}) \\
\text { and } w_{4}=700 \mathrm{~s}(25 \mathrm{~km})\end{array}$ & No periodicities found (Fig. 9c,d) \\
\hline \multirow[t]{4}{*}{$\begin{array}{l}\text { Generating } \\
\text { cells } \\
1-2 \mathrm{~km}\end{array}$} & & $\begin{array}{l}\text { at } d=3 / 4-\text { Turbulence likely, } \\
\text { because the Kolmogorov } 4 / 5 \text { law } \\
\text { is fulfilled }\left(a_{1}=2 b_{2} \text { see Table } 1\right)\end{array}$ \\
\hline & 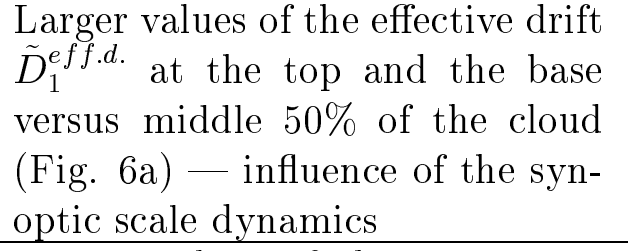 & $\begin{array}{l}\text { Larger values of the effective drift } \\
\tilde{D}_{1}^{\text {eff.d. }} \text { at the base versus middle } \\
50 \% \text { of the cloud (Fig. } 6 \mathrm{~b})- \text { in- } \\
\text { fluence of the synoptic scale dy- } \\
\text { namics }\end{array}$ \\
\hline & \multicolumn{2}{|c|}{$\begin{array}{l}\text { Larger values of the noice-induced drift } D_{1}^{n . i} \text { that represents the } \\
\text { stochasticity of the system in the middle } 50 \% \text { versus } \\
- \text { in both the upper and the lower - in both the upper } 33 \% \text { and the } \\
25 \% \text { (Fig. 6a) } \\
\text { This is in accordance with what is expected from the ice crystal } \\
\text { growth and deposition region. }\end{array}$} \\
\hline & \multicolumn{2}{|c|}{$\begin{array}{l}\text { Larger values of the noice-induced drift } \bar{D}_{1}^{n . i .} \text { in the middle } 50 \% \text { of } \\
\text { cirrus } A \text { versus } \tilde{D}_{1}^{n . i .} \text { in the middle } 50 \% \text { of cirrus } B \text {, which is in accor- } \\
\text { dance with intensive ice producing generating cells in } A \text { (Fig.6a,b) }\end{array}$} \\
\hline
\end{tabular}




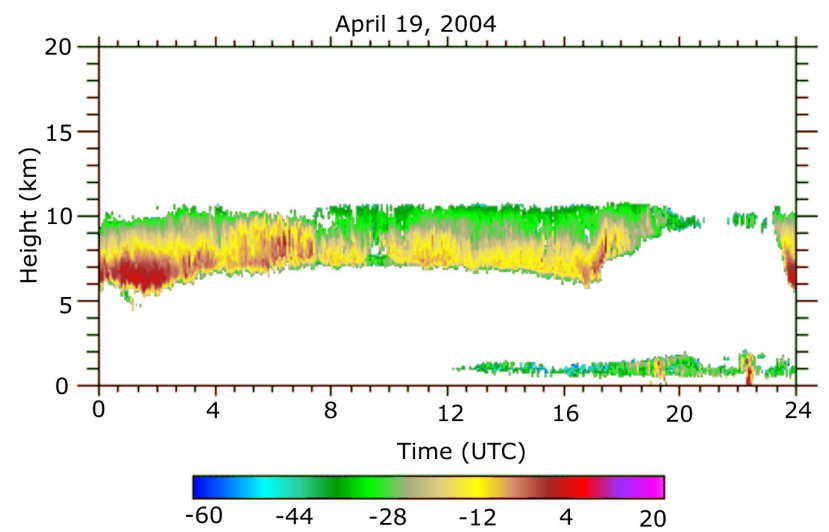

Figure 17: Radar reflectivity observations collected with the ARM ground-based millimeter wavelength cloud radar at the SGP facility near Lamont, OK on April 19, 2004. 